\title{
Semi-Global Solutions of Einstein Equations, Minkowskian Near Past Infinity
}

\author{
F. Cagnac and N. Noutchegueme \\ Faculté des Sciences, Université de Yaoundé, B.P. 812 Yaoundé, Cameroun
}

\begin{abstract}
On a universe homeomorphic to $\left.V^{T}=\right]-\infty, T\left[x \mathbb{R}^{3}\right.$, we prove the existence of solutions of Einstein equations, minkowskian near past infinity, if the sources are small enough for some norms. We prove that some of these solutions verify at least the positivity condition ("Weak energy condition") on some domains homeomorphic to $V^{T}$.
\end{abstract}

\section{Introduction}

In this paper we prove the existence of solutions of Einstein equations with sources in a universe homeomorphic to $\left.V^{T}=\right]-\infty, T\left[x R^{3}\right.$, such that hypersurfaces $\{t\} x R^{3}$ are spacelike and curves $]-\infty, T[x\{x\}$ are timelike, without any hypothesis of symmetry. We assume only the hypothesis of sources tending to 0 , if $t \rightarrow-\infty$, and "small" enough, for some norms, we shall define.

Such solutions represent universes with creation of matter and the question arises, whether they may possess physical meaning, particularly whether they may verify the positivity conditions. Concerning the condition $T^{\alpha \beta} X_{\alpha} X_{\beta} \geqq 0$ for any timelike vector field $X_{\alpha}$, where $T^{\alpha \beta}$ is the stress-energy tensor, that is the "weak energy condition" of Hawking and Ellis [11], we prove the existence of a solution, which verifies this positivity condition.

However, if the natural frame of $\left.V^{T}=\right]-\infty, T\left[x \mathbb{R}^{3}\right.$ is minkowskian as $t \rightarrow-\infty$, that is $g_{\alpha \beta}(t, x) \rightarrow \eta_{\alpha \beta}$, as $t \rightarrow-\infty$, where $\eta_{\alpha \beta}$ is the minkowskian metric, it is dubious that we can obtain solutions verifying the positivity condition on the whole of $V^{T}$.

We prove only the existence of solutions verifying the positivity condition on domains $D$, which, in a natural frame minkowskian as $t \rightarrow-\infty$, are defined by:

$$
\left.D=\left\{(t, x) \in R^{4} \mid t<T \text { and }\left|x-x_{0}\right|<A+\gamma(T-t)\right\}, \gamma>1\right\}
$$

or in a domain $D$ which is a union of such domains, spatially bounded for each value of $t$. But considering their topology and causal structure, such domains are isomorphic to a $V^{T}$. 
Do our solutions verify also the "dominant energy condition" of Hawking and Ellis? The answer is no! It can be said that their decay as $t \rightarrow-\infty$ is too great in order that such a condition can be verified.

The starting point of this paper is the thesis of Noutchegueme [1-4], who proves the existence of semi-global solutions of Einstein equations minkowskian near past infinity. This work was improved by Cagnac, Choquet-Bruhat, and Noutchegueme, who can prove the same results with not so strong conditions on the data [5].

However, the solutions were such that $g_{\alpha \beta}-\eta_{\alpha \beta}$ were square integrable functions on the space like hypersurfaces $\{t\} x R^{3}$; therefore they could only have a null ADM-mass and could not verify the positivity condition. After that, we proved the existence of solutions $g_{\alpha \beta}$ such that $g_{\alpha \beta}-\eta_{\alpha \beta}$ were locally square integrable functions on the space-like hypersurfaces, cancelling this difficulty. This was the object of two short notes $([6,7])$.

In this paper we give the first complete proof of the solutions in spaces of locally square integrable functions and we prove the existence of solutions verifying the positivity condition.

This paper is divided into three parts:

I. Choice of unknowns and equations

II. Solution of the equations

III. Positivity condition for the solutions.

\section{Choice of Unknowns and Equations}

A. The Problem. Einstein equations with harmonic coordinates and conservation equations can be written:

$$
\left\{\begin{array}{l}
-\frac{1}{2} g^{\lambda \mu} \partial_{\lambda \mu} g_{\alpha \beta}+h_{\alpha \beta}(g) \nabla g \otimes \nabla g=\varrho_{\alpha \beta}, \quad \alpha, \beta, \lambda, \mu=0, \ldots, 3, \\
\mid \nabla_{\alpha}\left(\varrho^{\alpha \beta}-\frac{1}{2} g^{\alpha \beta} \varrho_{\lambda}^{\lambda}\right)=0,
\end{array}\right.
$$

$g_{\alpha \beta}$ is the metric tensor,

$\varrho_{\alpha \beta}=T_{\alpha \beta}-\frac{1}{2} g_{\alpha \beta} T_{\lambda}^{\lambda}, T_{\alpha \beta}$ is the stress-energy tensor,

$h_{\alpha \beta}(g) \nabla g \otimes \nabla g$ means a sum of terms

$$
h_{\alpha \beta}^{\lambda \mu \nu, \varrho \sigma \tau}(g) \partial_{\lambda} g_{\mu \nu} \partial_{\varrho} g_{\sigma \tau},
$$

where the $h_{\alpha \beta}^{\lambda \mu \nu, \rho \sigma \tau}(g)$ are analytic functions of $g_{\lambda \mu}$ in the neighbourhood of $g_{\lambda \mu}=\eta_{\lambda \mu}$. (We shall also use this notation with vector functions other than $g$ ).

$\mid \nabla$ is the covariant derivative defined by $g$.

$$
\mid \begin{aligned}
& \text { We are seeking functions }\left(g_{\alpha \beta}, \varrho_{\alpha \beta}\right) \text { on } V^{T} \text {, verifying } \\
& \text { (I.1) and (I.2) such that } g_{\alpha \beta} \rightarrow \eta_{\alpha \beta} \text { when } t \rightarrow-\infty \text {. }
\end{aligned}
$$

Conversely, if functions $\left(g_{\alpha \beta}, \varrho_{\alpha \beta}\right)$ on $V^{T}$ verify (I.1) and (I.2) they define a solution of Einstein equations with sources if the natural coordinates of $V^{T}$ are harmonic for $g$. That is, if $g$ verifies the gauge conditions:

$$
\Phi^{\lambda} \equiv \Gamma_{\alpha \beta}^{\lambda} g^{\alpha \beta}=0 .
$$


( $\Gamma_{\alpha \beta}^{\lambda}$ are the coefficients of the connexion defined by $g$ ); when we have obtained solutions $\left(g_{\alpha \beta}, g_{\alpha \beta}\right)$ of (I.1, I.2), we must consider whether $g_{\alpha \beta}$ verifies (I.3).

B. Choice of the Unknowns. If we assume given the $\varrho^{i j}(i, j=1,2,3)$ and the $g_{\alpha \beta}$, we can prove that the system (I.2) is a hyperbolic system for the unknowns $\varrho^{0 \alpha}$ $(\alpha=0, \ldots, 3)$ (cf. [5]).

However this system becomes "almost" an hyperbolic symmetric system, and, therefore, is easier to study if we do not take as unknowns the components of $\varrho$ in the natural frame of $V^{T}$, but the components of $\varrho$ in a moving orthonormal frame $e=\left(e_{A}^{\alpha}\right)(A=0, \ldots, 3)$.

Hence we proceed among the following lines: In place of the tensor $g$, we take as unknowns an orthonormal moving frame $e=\left(e_{A}^{\alpha}\right)$, such that $e_{A}^{\alpha} \rightarrow \delta_{A}^{\alpha}$ as $t \rightarrow-\infty . g$ is linked with $e$ by:

$$
g_{\alpha \beta}=e_{\alpha}^{A} e_{\beta}^{B} \eta_{A B},
$$

where $\left(e_{\alpha}^{A}\right)$ is the inverse matrix of $\left(e_{A}^{\alpha}\right)$.

The equations (I.2) can be written:

$$
\left\{\begin{array}{ll}
\left|\nabla_{0} \varrho^{00}-\frac{1}{2} \eta_{C D}\right| \nabla_{0} \varrho^{C D}+\sum_{I} \mid \nabla_{I} \varrho^{0 I}=0, & C, D=0, \ldots, 3 \\
\left|\nabla_{0} \varrho^{0 J}+\sum_{I}\right| \nabla_{I} \varrho^{I J}+\frac{1}{2} \eta_{C D} \mid \nabla_{J} \varrho^{C D}=0, & I, J=1,2,3
\end{array} .\right.
$$

Hence, putting $\varrho^{0 A}=V^{A}, \varrho^{I J}=\phi^{I J}$, and considering that the coefficients $\gamma_{A B}^{C}$ of the connection are linear in $\partial_{\lambda} e_{\alpha}^{A}$ with coefficients, which are products of some $e_{\alpha}^{A}$ and $e_{A}^{\alpha}$, we obtain

$$
\left\{\begin{array}{l}
\frac{1}{2} \partial_{0} V^{0}+\partial_{I} V^{I}=f^{0}, \\
\frac{1}{2} \partial_{J} V^{0}+\partial_{0} V^{J}=f^{J},
\end{array} \quad\left(\partial_{A} V^{B}=e_{A}^{\alpha} \partial_{\alpha} V^{B}\right)\right.
$$

where the $f^{A}$ can be written

$$
f^{A}=k(e) \nabla e \otimes V+k(e) \nabla e \otimes \phi+k(e) \nabla \phi,
$$

$k(e)$, analytic functions of the $e_{\alpha}^{A}$, in a neighbourhood of $e_{\alpha}^{A}=\delta_{\alpha}^{A}$.

Considering (I.4), Eqs. (I.1) are written

$$
-\frac{1}{2} g^{\lambda \mu}(e) \eta_{A B}\left(e_{\beta}^{B} \partial_{\lambda \mu} e_{\alpha}^{A}+e_{\alpha}^{A} \partial_{\lambda \mu} e_{\beta}^{B}\right)+h_{\alpha \beta}^{\prime}(e) \nabla e \otimes \nabla e=\varrho_{\alpha \beta},
$$

where the $h_{\alpha \beta}^{\prime}$ are symmetric in $\alpha, \beta$.

We impose on the $e_{A}^{\alpha}$ the gauge conditions:

$$
\Phi_{\alpha \beta} \equiv \eta_{A B} g^{\lambda \mu}(e)\left(e_{\alpha}^{A} \partial_{\lambda \mu} e_{\beta}^{B}-e_{\beta}^{A} \partial_{\lambda \mu} e_{\alpha}^{B}\right)=0
$$

Then, Eq. (I.6) become:

$$
-g^{\lambda \mu}(e) \eta_{A B} e_{\beta}^{B} \partial_{\lambda \mu} e_{\alpha}^{A}+h_{\alpha \beta}^{\prime}(e) \nabla_{e} \otimes \nabla_{e}=\varrho_{\alpha \beta} .
$$

Conversely, if $e_{\alpha}^{A}$ verify (I.8), the fact that $h_{\alpha \beta}^{\prime}$ and $\varrho_{\alpha \beta}$ are symmetric in $\alpha, \beta$ implies that the gauge conditions (I.7) are satisfied.

The system (I.8) is equivalent to the following system, obtained by linear combinations:

$$
g^{\lambda \mu}(e) \partial_{\lambda \mu} e_{\alpha}^{A}-h_{\alpha \beta}^{\prime}(e) \nabla_{e} \otimes \nabla_{e} e_{C}^{\beta} \eta^{A C}=\varrho_{\alpha \beta} e_{C}^{\beta} \eta^{A C}
$$


We choose as unknowns:

$$
u_{\alpha}^{A}=e_{\alpha}^{A}-\delta_{\alpha}^{A}, \quad V^{A}=\varrho^{0 A} .
$$

The components $\phi^{I J}=e^{I J}$ are assumed given.

Finally, we obtained the following system of equations for the unknowns $u=\left(u_{\alpha}^{A}\right)$ and $V=\left(V^{A}\right)$ :

$$
\left\{\begin{array}{l}
g^{\lambda \mu}(u) \partial_{\lambda \mu} u+h(u) \nabla u \otimes \nabla u=l(u, V, \phi) \\
L(u) V=f(u, V, \phi)
\end{array}\right.
$$

$L(u)$ is the differential matrix operator defined by the left-hand side of (I.5),

$$
\begin{gathered}
l(u, V, \phi)=k(u) V+k(u) \phi, \\
f(u, V, \phi)=k(u) \nabla u \otimes V+k(u) \nabla u \otimes \phi+k(u) \nabla \phi .
\end{gathered}
$$

$g^{\lambda \mu}(u), h(u)$, and the functions $k(u)$ are analytic functions of $u_{\alpha}^{A}$ in the neighbourhood of $u_{\alpha}^{A}=0$. A solution $(u, V)$ of (I.10) will define a solution of Einstein equations, if the gauge conditions (I.3) are satisfied by the solution.

\section{Solution of Equations}

Notations and Results.

$$
\begin{aligned}
& \left.V^{T}=\right]-\infty, T\left[x R^{3},\right. \\
& \left(x^{\alpha}\right)=(t, x) \in V^{T}, \quad \alpha=0, \ldots, 3, \quad t=x^{0}, \quad x=\left(x^{i}\right), \quad i=1, \ldots, 3, \\
& |x|=\left(\Sigma\left(x^{i}\right)^{2}\right)^{1 / 2}, \\
& u=\left(u_{\alpha}^{A}\right)=u^{I} \quad \text { (with a unique index for simplification), } \\
& D^{\lambda} u^{I}=\frac{\partial^{|\lambda|} u^{I}}{\partial x_{0}^{\lambda_{0}} \ldots \partial x_{3}^{\lambda_{3}}} \text {, where } \lambda=\left(\lambda_{0}, \ldots, \lambda_{3}\right),|\lambda|=\lambda_{0}+\ldots+\lambda_{3} \text {, } \\
& \left|\nabla^{i} u\right|=\left(\sum_{I,|\lambda|=i}\left|D^{\lambda} u^{I}\right|^{2}\right)^{1 / 2}, \quad \text { if } \Omega_{t} \subset\{t\} x R^{3}, \\
& \left\|\nabla^{i} u\right\|_{\Omega_{t}}=\left(\int_{\Omega_{t}}\left|\nabla^{i} u\right|^{2} d x\right)^{1 / 2}, \quad d x=d x^{1} d x^{2} d x^{3} .
\end{aligned}
$$

If $i=1$, we write simply $|\nabla u|=\left|\nabla^{1} u\right|,\|\nabla u\|_{\Omega_{t}}=\left\|\nabla^{1} u\right\|_{\Omega_{t}}$.

If $u, v, w$ are vector functions, $k(u) v \otimes w$ means a vector function, whose components are sums of terms $k\left(u^{I}\right) v^{A} w^{B} . k(u)$ may represent different functions at each use of this symbol, even in the same line. Similarly, $C$ will represent different constants.

Domains $\Omega$. There exists an $\varepsilon_{0}>0$ such that $|u|=\operatorname{Sup}\left|u^{I}\right|<\varepsilon_{0}$, implies: $g^{\alpha \beta}(u) \partial_{\alpha \beta}$ is a regularly hyperbolic operator and $g^{\alpha \beta}(u), h_{\alpha \beta}(u)$ in (I.10) and the $k(u)$ in (I.11), (I.12) are analytic functions for $|u|<\varepsilon_{0}$.

Then, there exists a $\gamma>1$, such that, if $|u|<\varepsilon_{0}$, the characteristic cones of $g^{\alpha \beta}(u) \partial_{\alpha \beta}$ are contained in the cone $|x|<\gamma t$. For each $T \in R$ and $a \in R^{3}$, we define a domain $\Omega(T, a)$ by:

$$
\Omega(T, a)=\left\{(t, x) \in R^{4} \mid t<T \text { and }|x-a|<1+\gamma(T-t)\right\} .
$$


Each domain, for any $T$ and $a$, will be called "a domain $\Omega$ ". We have

$$
V^{T}=\bigcup_{a \in R^{3}} \Omega(T, a) .
$$

So chosen, the domains $\Omega$ are Causal domains for $g^{\alpha \beta}(u) \partial_{\alpha \beta}$ if $|u|<\varepsilon_{0}$. We put:

$$
\forall t \in]-\infty, T\left[, \quad \Omega_{t}(T, a)=\Omega(T, a) \cap\{t\} x R^{3} .\right.
$$

The $\Omega_{t}$ 's are balls in $R^{3}$ with a radius greater than 1 , in such a way that the inequalities of Sobolev can be verified with the same constants in all the $\Omega_{t}(T, a)$.

Function Spaces and Norms. For any domain $\Omega$, we define

$$
C^{\infty}(\Omega)=\left\{f \in C^{\infty}(\bar{\Omega}) ; \text { there exists } t_{0} \text { such that } f=0 \text { if } t \leqq t_{0}\right\},
$$

$E_{k}(\Omega)$ is the closure of $C^{\infty}(\Omega)$ in the norm

We put $q_{\Omega, 3}=q_{\Omega}$.

$$
q_{\Omega, k}(f)=\operatorname{Sup}_{t<T} \sum_{i=0}^{k}\left\|\nabla^{i} f\right\|_{\Omega_{t}} .
$$

$$
\begin{aligned}
& \tilde{E}(\Omega)=\left\{f \in E_{3}(\Omega) \text { and } \tilde{q}_{\Omega}(f)=q_{\Omega}(\tilde{f})+\int_{-\infty}^{T}(T-t+1)\right. \\
&\left.\quad \times \sup _{\tau<t}\left(\sum_{i=0}^{3}\left\|\nabla^{i} f\right\|_{\Omega_{T}}\right) d t<+\infty\right\} . \\
& \tilde{E}^{*}(\Omega)=\left\{f \in \tilde{E}(\Omega) \text { and } \tilde{q}_{\Omega}^{*}(f)=\tilde{q}_{\Omega}(f)+\underset{t<T}{\text { Ess } \left.\operatorname{Sup}\left\|\nabla^{4} f\right\|_{\Omega_{t}}<+\infty\right\} .}\right. \\
& \mathscr{L}(\Omega)=\left\{f \text { measurable on } \Omega \text { and } p_{\Omega}(f)=\int_{-\infty}^{T}\left\{(T-t+1)^{3}\|f\|_{\Omega_{t}}\right.\right. \\
&\left.\left.+\sum_{k=1}^{3}(T-t+1)^{4-k}\left\|\nabla^{k} f\right\|_{\Omega_{t}}\right\} d t<+\infty\right\} .
\end{aligned}
$$

On $\mathscr{L}(\Omega)$, we shall use also the norm

$$
\begin{aligned}
p_{\Omega}^{1}(f)= & \int_{-\infty}^{T}\left\{(T-t+1)^{3}\|f\|_{\Omega_{t}}+\sum_{k=1}^{2}(T-t+1)^{4-k}\left\|\nabla^{k} f\right\|_{\Omega_{t}}\right\} d t . \\
\hat{\mathscr{L}}(\Omega)= & \left\{f \text { measurable on } \Omega \text { and } \hat{p}_{\Omega}(f)=\int_{-\infty}^{T}\left\{(T-t+1)^{5}\|f\|_{\Omega_{t}}\right.\right. \\
& \left.\left.+\sum_{k=1}^{4}(T-t+1)^{6-k}\left\|\nabla^{k} f\right\|_{\Omega_{t}}\right\} d t<+\infty\right\} .
\end{aligned}
$$

$E_{k}(\Omega), \tilde{E}(\Omega), \widetilde{E}^{*}(\Omega), \mathscr{L}(\Omega), \hat{\mathscr{L}}(\Omega)$ are Banach spaces, $C^{\infty}(\Omega)$ is dense in $\mathscr{L}(\Omega)$.

The Main Theorem. There exist constants $r_{0}$ and $\hat{r}$ such that if for a domain $\Omega$, $\hat{p}_{\Omega}(\phi)<\hat{r}$, the system (I.10) has a unique solution $(u, V)$ in this domain $\Omega$, such that

$$
\tilde{q}_{\Omega}^{*}(u)<r_{0} \quad \text { and } \quad V \in E_{3}(\Omega) .
$$

This solution satisfies the gauge conditions (I.3) and defines a solution of Einstein equations with sources, minkowskian near past infinity. 
Corollary. If $D$ is a union of domains $\Omega$ 's, and if, for each of these $\Omega$ 's, $\hat{p}_{\Omega}(\phi)<\hat{r}$. $\Omega$ 's.

The system (I.10) has a unique solution $(u, V)$ in D verifying $\left(^{*}\right)$ for each of these

This solution defines a solution of Einstein equations with sources, minkowskian near past infinity.

This corollary can be applied to $V^{T}=\bigcup_{a \in R^{3}} \Omega(T, a)$ if $\hat{p}_{\Omega}(\phi)<\hat{r}$ for all $\Omega(T, a)$, $a \in R^{3}$.

II.A. The System

$$
g^{\alpha \beta}(u) \partial_{\alpha \beta}^{2} u^{I}=\varrho^{I}
$$

1. Basic Inequalities for (II.A.1)

Proposition 1. Let $u \in C^{\infty}(\Omega)$ be a solution of (II.A.1) such that $|u|<\varepsilon_{0}$. Then u verifies for $t<T$ the inequalities:

a)

$$
\begin{gathered}
\|\nabla u\|_{\Omega_{t}} \leqq \Phi_{1}\left(P_{u, \Omega}\right) \int_{-\infty}^{t}\|\varrho\|_{\Omega_{\tau}} d \tau \\
\left\|\nabla^{2} u\right\|_{\Omega_{t}} \leqq \Phi_{2}\left(P_{u, \Omega}\right) \int_{-\infty}^{t}\|\nabla \varrho\|_{\Omega_{\tau}} d \tau
\end{gathered}
$$

b)

c)

$$
\begin{gathered}
\left\|\nabla^{3} u\right\|_{\Omega_{t}} \leqq \Phi_{3}\left(P_{u, \Omega}, M_{u, \Omega}, N_{u, \Omega}\right) \int_{-\infty}^{t}\left\{(t-\tau)\|\nabla \varrho\|_{\Omega_{\tau}}+\left\|\nabla^{2} \varrho\right\|_{\Omega_{\tau}}\right\} d \tau \\
\left\|\nabla^{4} u\right\|_{\Omega_{t}} \leqq \\
\Phi_{4}\left(P_{u, \Omega} ; M_{u, \Omega}, N_{u, \Omega}\right) \int_{-\infty}^{t}\left\{(t-\tau+1)^{2}\|\nabla \varrho\|_{\Omega_{\tau}}\right. \\
\left.+(t-\tau)\left\|\nabla^{2} \varrho\right\|_{\Omega_{\tau}}+\left\|\nabla^{3} \varrho\right\|_{\Omega_{\tau}}\right\} d \tau
\end{gathered}
$$

where $\Phi_{i}(i=1,2,3,4)$ are continuous and increasing functions of:

$$
P_{u, \Omega}=\int_{-\infty}^{T} \operatorname{Sup}_{\Omega_{t}}|\nabla u| d t ; M_{u, \Omega}=\operatorname{Sup}_{\Omega}|\nabla u| ; N_{u, \Omega}=\operatorname{Sup}_{\Omega}\left|\nabla^{2} u\right|
$$

Proof. Multiply (II.A.1) by $\partial_{0} u$ and integrate the equality over

$$
A=\Omega \cap\left\{t_{0}<\tau<t\right\},
$$

where $t_{0}$ is such that $u=0$ for $t \leqq t_{0}$. Stokes' formula yields:

$$
\begin{aligned}
& \int_{F}\left\{\frac{n_{0}}{2}\left(g^{00}\left(\partial_{0} u\right)^{2}-g^{i j} \partial_{i} u \partial_{j} u\right)+n_{i}\left(g^{0 i}\left(\partial_{0} u\right)^{2}+g^{i j} \partial_{0} u \partial_{j} u\right)\right\} d S \\
&=\int_{A}\left\{\varrho \partial_{0} u+\left(\partial_{0} u\right)^{2}\left[\partial_{i} g^{0 i}+\frac{1}{2} \partial_{0} g^{00}\right]\right. \\
&\left.+\partial_{0} u \partial_{i} u \partial_{j} g^{i j}-\frac{1}{2} \partial_{i} u \partial_{j} u \partial_{0} g^{i j}\right\} d \tau d x,
\end{aligned}
$$

where $\left(n_{\alpha}\right)$ is the unit outward normal to the boundary $F$ of $A$, and $d S$ the measure element on $F$. $F$ is made of 3 parts:

$F_{1}$ on $\tau=t ; F_{2}$ on $\tau=t_{0} ; F_{3}$ on the "lateral boundary" of $\Omega$. 


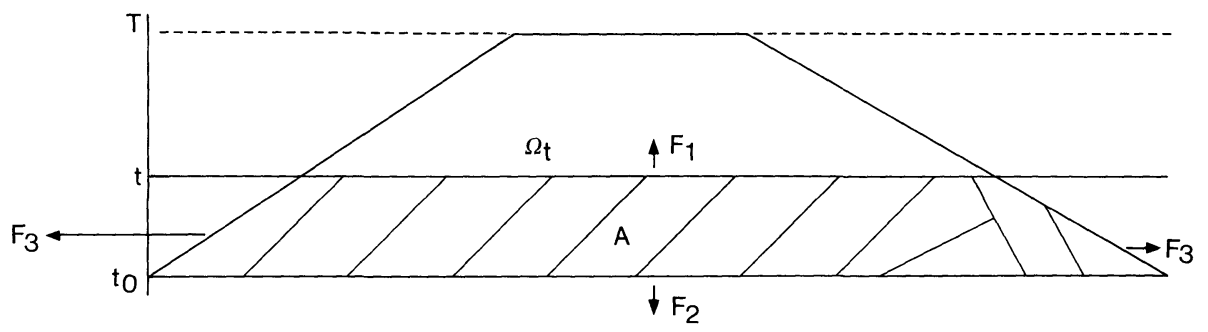

We have $\int_{F_{2}}=0$.

The hypothesis $|u|<\varepsilon_{0}$ implies that there exists a constant $C>0$ such that:

$$
\int_{F_{1}}=\int_{\Omega_{t}} \frac{1}{2}\left(g^{00}\left(\partial_{0} u\right)^{2}-g^{i j} \partial_{i} u \partial_{j} u\right) d x \geqq C\|\nabla u\|_{\Omega_{t}}^{2} .
$$
Now on $F_{3}$ we have $d S=\frac{d x}{n_{0}}$, and in the left-hand side of $(*)$ the integral over $F_{3}$ can
be written as:

$$
I=\int_{F_{3}} \frac{1}{n_{0}^{2}}\left\{\left(\partial_{0} u\right)^{2} \frac{1}{2} g^{\alpha \beta} n_{\alpha} n_{\beta}-\frac{1}{2} g^{i j}\left(n_{0} \partial_{i} u-n_{i} \partial_{0} u\right)\left(n_{0} \partial_{j} u-n_{j} \partial_{0} u\right)\right\} d x .
$$

But $F_{3}$ is spatial; so we have $g^{\alpha \beta} n_{\alpha} n_{\beta} \geqq 0$. On the other hand, the form $g^{i j} X_{i} X_{j}$ is negative definite. This implies $I \geqq 0$. We then deduce from $(*)$ the inequality:

$$
\|\nabla u\|_{\Omega_{t}}^{2} \leqq C \int_{t_{0}}^{t} d \tau \int_{\Omega_{\tau}}\left\{\sum_{\alpha, \lambda, \mu}\left(\operatorname{Sup}_{\Omega_{\tau}}\left|\partial_{\alpha} g^{\lambda \mu}\right|\right)|\nabla u|^{2}+|\varrho||\nabla u|\right\} d x .
$$

Now by chain rule, we have:

$$
\partial_{\alpha} g^{\lambda \mu}=\sum_{I} \frac{\partial g^{\lambda \mu}}{\partial u^{I}} \partial_{\alpha} u^{I}
$$

Since the $\frac{\partial g^{\lambda \mu}}{\partial u^{I}}$ are bounded, we deduce from $(* *)$, using Schwarz' inequality:

$$
\|\nabla u\|_{\Omega_{t}}^{2} \leqq C \int_{t_{0}}^{t}\left\{\left(\operatorname{Sup}_{\Omega_{\tau}}|\nabla u|\right)\|\nabla u\|_{\Omega_{\tau}}^{2}+\|\varrho\|_{\Omega_{\tau}}\|\nabla u\|_{\Omega_{\tau}}\right\} d \tau
$$

and Gronwall's lemma yields:

$$
\|\nabla u\|_{\Omega_{t}} \leqq \frac{C}{2} \exp \left[\frac{C}{2} \int_{-\infty}^{T} \operatorname{Sup}|\nabla u| d t\right] \cdot \int_{-\infty}^{t}\|\varrho\|_{\Omega_{\tau}} d \tau .
$$

This proves the inequality a).

We obtain the inequalities b), c), d) by the same method applied on equations deduced from (II.A.1) by successive derivations up to order 3 with respect to $x^{\lambda}$. This ends the proof of Proposition 1.

Our next purpose is to deduce from these inequalities, some other inequalities with absolute constants. 
Proposition 2. Given arbitrary positive constants $M_{0}, N_{0}$, there exists a number $r>0$ such that, if $\varrho$ satisfies the condition:

$$
\int_{-\infty}^{T}\left\{(T-t+1)\|\varrho\|_{\Omega_{t}}+\sum_{i=1}^{3}(T-t+1)^{3-i}\left\|\nabla^{i} \varrho\right\|_{\Omega_{t}}\right\} d t<r .
$$

Then, if $u \in \underline{C}^{\infty}(\Omega)$ and verifies (II.A.1):

$\left.1^{\circ}\right)$ We have:

$$
M_{u, \Omega}<M_{0} ; \quad N_{u, \Omega}<N_{0} ; \quad P_{u, \Omega}<\varepsilon_{0} ; \quad|u|<\varepsilon_{0} .
$$

$\left.2^{\circ}\right)$ There exists $K_{0}>0$ such that $u$ verifies the inequalities:

(ع) $\left\|\nabla^{4} u\right\|_{\Omega_{t}} \leqq K_{0} \int_{-\infty}^{t}\left\{(t-\tau+1)^{2}\|\nabla \varrho\|_{\Omega_{\tau}}+(t-\tau)\left\|\nabla^{2} \varrho\right\|_{\Omega_{\tau}}+\left\|\nabla^{3} \varrho\right\|_{\Omega_{\tau}}\right\} d \tau$.

Proof. There exists a Sobolev constant $C>0$ such that:

$$
\operatorname{Sup}_{\Omega_{t}}|\nabla u| \leqq C \sum_{i=1}^{3}\left\|\nabla^{i} u\right\|_{\Omega_{t}} ; \quad \operatorname{Sup}_{\Omega_{t}}\left|\nabla^{2} u\right| \leqq C \sum_{i=2}^{4}\left\|\nabla^{i} u\right\|_{\Omega_{t}} .
$$

Suppose we have:

$$
P_{u, \Omega}<\varepsilon_{0}, \quad M_{u, \Omega}<M_{0}, \quad N_{u, \Omega}<N_{0} .
$$

This will imply, using Proposition 1:

$$
\left\{\begin{aligned}
M_{u, \Omega} \leqq & C \psi_{1}\left(\varepsilon_{0}, M_{0}, N_{0}\right) \int_{-\infty}^{T}\left\{\|\varrho\|_{\Omega_{t}}+(T-t+1)\|\nabla \varrho\|_{\Omega_{t}}+\left\|\nabla^{2} \varrho\right\|_{\Omega_{t}}\right\} d t \\
P_{u, \Omega} \leqq & C \psi_{2}\left(\varepsilon_{0}, M_{0}, N_{0}\right) \int_{-\infty}^{T}\left\{(T-t)\|\varrho\|_{\Omega_{t}}+(T-t+1)^{2}\|\nabla \varrho\|_{\Omega_{t}}\right. \\
& \left.+(T-t)\left\|\nabla^{2} \varrho\right\|_{\Omega_{t}}\right\} d t \\
N_{u, \Omega} \leqq & C \psi_{3}\left(\varepsilon_{0}, M_{0}, N_{0}\right) \int_{-\infty}^{T}\left\{(T-t+1)^{2}\|\nabla \varrho\|_{\Omega_{t}}\right. \\
& \left.+(T-t+1)\left\|\nabla^{2} \varrho\right\|_{\Omega_{t}}+\left\|\nabla^{3} \varrho\right\|_{\Omega_{t}}\right\} d t .
\end{aligned}\right.
$$

One then chooses $r$ in such a way that condition(II.A.2) implies that the right-hand sides in (2) be respectively less than $M_{0}^{\prime}<M_{0}, \varepsilon_{0}^{\prime}<\varepsilon_{0}, N_{0}^{\prime}<N_{0}$.

Under these conditions, (1) implies:

$$
M_{u, \Omega}<M_{0}^{\prime}, \quad P_{u, \Omega}<\varepsilon_{0}^{\prime}, \quad N_{u, \Omega}<N_{0}^{\prime} .
$$

But when $T \rightarrow-\infty,(3)$ is verified, since $u \in \underline{C^{\infty}}(\Omega)$. Now $P_{u, \Omega}, M_{u, \Omega}, N_{u, \Omega}$ are continuous functions of $T$. So the fact that (1) $\Rightarrow(3)$ shows that $\left(P_{u, \Omega}, M_{u, \Omega}, N_{u, \Omega}\right)$ 
cannot go outside the connected domain of $\mathbb{R}^{3}$ defined by (3). This proves (II.A.3). The inequalities (II.A.4) are direct consequences of a), b), c), d) Proposition 1. This ends the proof of Proposition 2.

Note. In all the following, $r$ will always stand for this number in (II.A.2).

Proposition 3. $1^{\circ}$ ) If $|u|<\varepsilon_{0}, P_{u, \Omega}<\varepsilon_{0}, v \in E_{1}(\Omega)$, and $\left|\nabla^{2} v\right|$ is locally square integrable, then if

$$
g^{\alpha \beta}(u) \partial_{\alpha \beta}^{2} v=\varrho,
$$

$v$ verifies the inequalities (II.A.4), ( $\alpha$ ) and ( $\beta$ ).

$\left.2^{\circ}\right)$ Moreover, if $M_{u, \Omega}<M_{0}, N_{u, \Omega}<N_{0}$ and if $v \in E_{3}(\Omega)$ with $\left|\nabla^{4} v\right|$ locally square integrable, then $v$ verifies the inequalities (II.A.4), $(\gamma)$ and $(\delta)$.

Proof. Is straightforward the proof of Proposition 1. One just has to distinguish between what depends on $g^{\alpha \beta}$ and what depends on $v$.

\section{Solutions of (II.A.1)}

Theorem 1. $\forall \varrho \in C^{\infty}(\Omega)$ satisfying (II.A.2), (II.A.1) has a unique solution $u$ in $\underline{C}^{\infty}(\Omega)$. This solution verifies (II.A.3) and (II.A.4).

Proof. It is based on Leray-Dionne Results [8-9], and is similar to the proof given in [3], Sect. III, Proposition III. For the existence of the solution, $\varrho$ is then replaced by $\tilde{\varrho} \phi_{\Omega}$, where $\tilde{\varrho} \in C^{\infty}\left(\bar{V}_{4}^{T}\right)$ and $\left.\tilde{\varrho}\right|_{\Omega}=\varrho$, while $\phi_{\Omega}$ is a suitable truncating function.

Unicity is obtained using Gronwall's Lemma.

Theorem 2 [Existence and unicity of Solutions in $\left.E_{3}(\bar{\Omega})\right]$. Let $B(r)$ $=\left\{\varrho \in \mathscr{L}_{\Omega} \mid p_{\Omega}(\varrho) \leqq r\right\}$ and $\varrho \in B(r)$. Then

$\left.1^{\circ}\right)$ the system

$$
g^{\alpha \beta}(u) \partial_{\alpha \beta}^{2} u^{I}=\varrho^{I}
$$

has, in the sense of distributions, a unique solution $u$ in $E_{3}(\Omega)$ such that:

$$
P_{u, \Omega}<\varepsilon_{0} .
$$

$\left.2^{\circ}\right)$ This solution has the following properties:

$u$ has $4^{\text {th }}$ derivatives, in the sense of distributions, which are, for almost every $t \in]-\infty, T[$, locally square integrable and:

$$
\left.\begin{array}{c}
\text { Ess Sup }\left\|\nabla^{4} u\right\|_{\Omega_{t}} \leqq K_{0} p_{\Omega}(\varrho) . \\
|u|<\varepsilon_{0} ; \quad|\nabla u|<M_{0} ; \quad\left|\nabla^{2} u\right|<N_{0} . \\
\|u\|_{\Omega_{t}} \leqq K_{0} \int_{-\infty}^{t}(t-\tau)\|\varrho\|_{\Omega_{\tau}} d \tau \\
\|\nabla u\|_{\Omega_{\tau}} \leqq K_{0} \int_{-\infty}^{t}\|\varrho\|_{\Omega_{\tau}} d \tau \\
\left\|\nabla^{2} u\right\|_{\Omega_{\tau}} \leqq K_{0} \int_{-\infty}^{t}\|\nabla \varrho\|_{\Omega_{\tau}} d \tau \\
\left.\left\|\nabla^{3} u\right\|_{\Omega_{t}} \leqq K_{0} \int_{-\infty}^{t}\{t-\tau)\|\nabla \varrho\|_{\Omega_{\tau}}+\left\|\nabla^{2} \varrho\right\|_{\Omega_{\tau}}\right\} d \tau .
\end{array}\right\}
$$


Remark. (II.A.6) and (II.A.8) imply

$$
\left\|\nabla^{i} u\right\|_{\Omega_{t}}<K_{0} r, \quad i=0,1, \ldots, 4
$$

Proof. Due to the density of $C^{\infty}(\Omega)$ in $\mathscr{L}_{\Omega}$, one approaches $\varrho$ by a sequence $\left(\varrho_{n}\right) \subset B(r) \cap \underline{C^{\infty}}(\Omega)$. The solutions $u_{n}$ of (II.A.1) given by Theorem 1 verify

$$
g^{\alpha \beta}\left(u_{n}\right) \partial_{\alpha \beta}^{2}\left(u_{n}-u_{m}\right)=\varrho_{n}-\varrho_{m}+\left[g^{\alpha \beta}\left(u_{m}\right)-g^{\alpha \beta}\left(u_{n}\right)\right] \partial_{\alpha \beta}^{2} u_{m} .
$$

We apply Proposition $\left.3,2^{\circ}\right)$ to $v=u_{n}-u_{m}$ and we use the fact that $p_{\Omega}\left(\varrho_{n}\right)<r$ to show that $\left(u_{n}\right)$ is a Cauchy sequence in the Banach space $E_{3}(\Omega)$, which converges to a weak solution $u$ of (II.A.1).

The inequality (II.A.4) $(\varepsilon)$ verified by $u_{n}$ and $\varrho_{n}$ implies that

$$
\operatorname{Sup}_{n} \operatorname{Sup}_{t<T}\left\|\nabla^{4} u_{n}\right\|_{\Omega_{t}} \leqq K_{0} r .
$$

Now, in a similar way to [3], Sect. VI, Lemma 3, one shows that the space:

$$
Q_{0}(\Omega)=\left\{f \text { measurable on } \Omega,\|f\|_{Q_{0}}=\underset{t<T}{\operatorname{Ess~Sup~}}\|f\|_{\Omega_{t}}<\infty\right\} .
$$

is the dual of the Banach space:

$$
F_{0}(\Omega)=\left\{g \text { measurable on } \Omega,\|g\|_{F_{0}}=\int_{-\infty}^{T}\|g\|_{\Omega_{\mathrm{t}}} d t<\infty\right\} .
$$

(II.A.6) is then a consequence of (1) and of the weak compacity of balls in $Q_{0}(\Omega)$. (II.A.8) is obtained from inequalities (II.A.4) $(\alpha)$ to ( $\delta$ ), on $u_{n}$ and $\varrho_{n}$, using the limiting process. (II.A.7) is a consequence of (II.A.8) and the construction of $r$. Unicity is obtained from Proposition $3,1^{\circ}$ ).

B. The System $L(u) V=f(u, V, \phi)$. We assume that $u$ and $\phi$ are given and $u$ verifies (II.A.5, 7, 9).

1. Estimates for the Solutions $V$. We first assume $V$ and $\phi$ in $\underline{C^{\infty}}(\Omega)$, null for $t \leqq t_{0}$ and satisfying

$$
\begin{aligned}
& \frac{1}{2} \partial_{0} V^{0}+\partial_{I} V^{I}=f^{0}, \\
& \frac{1}{2} \partial_{J} V^{0}+\partial_{0} V^{J}=f^{J},
\end{aligned} \quad\left(\partial_{A} V=e_{A}^{\alpha} \partial_{\alpha} V\right)
$$

From (II.B.1) we deduce:

$$
\partial_{0}\left[\frac{1}{4}\left(V^{0}\right)^{2}+\sum_{J}\left(V^{J}\right)^{2}\right]+\partial_{I}\left(V^{0} V^{I}\right)=f^{0} V^{0}+\sum_{J} 2 f^{J} V^{J} .
$$

If we write the derivatives in the natural frame of $V^{T}$, we obtain

$$
\begin{aligned}
& \partial_{0} {\left[\frac{1}{4}\left(V^{0}\right)^{2}+\sum_{J}\left(V^{J}\right)^{2}+k^{0}(u) V \otimes V\right]+\partial_{i}\left(V^{0} V^{i}+k^{i}(u) V \otimes V\right) } \\
&=f^{0} V^{0}+\sum_{J} 2 f^{J} V^{J}+k(u) V u \otimes V \otimes V,
\end{aligned}
$$

where the $k^{\lambda}(u)$ are some $e_{A}^{\alpha}-\delta_{A}^{\alpha}$. Therefore they verify estimates:

$$
\left|k^{\lambda}(u)\right| \leqq C|u|
$$


We integrate (II.B.2) on $\Omega \cap\left\{t_{0} \leqq \tau \leqq t\right\}$; we obtain:

$$
\begin{gathered}
\int_{F}\left\{n_{0}\left[\frac{1}{4}\left(V^{0}\right)^{2}+\sum_{j}\left(V^{j}\right)^{2}+k^{0}(u) V \otimes V\right]+n_{i}\left[V^{0} V^{i}+k^{i}(u) V \otimes V\right]\right\} d S \\
=\int_{t_{0}}^{t} d \tau \int_{\Omega_{\tau}}\left\{f^{0} V^{0}+\sum_{J} 2 f^{J} V^{J}+k(u) \nabla u \otimes V \otimes V\right\} d x .
\end{gathered}
$$

As in part II.A, $F=F_{1} \cup F_{2} \cup F_{3}$; and $\int_{F_{2}}=\int_{\Omega_{t_{0}}}=0$,

$$
\int_{F_{1}}=\int_{\Omega_{t}}\left(\frac{1}{4}\left(V^{0}\right)^{2}+\sum_{J}\left(V^{j}\right)^{2}+k^{0}(u) V \otimes V\right) d x .
$$

Considering (II.B.3) we have

$$
\left|\int_{\Omega_{t}} k^{0}(u) V \otimes V d x\right| \leqq C \operatorname{Sup}_{\Omega_{t}}|u|\|\nabla\|_{\Omega_{t}}^{2} \leqq C \operatorname{Sup}_{\Omega_{t}}|u| 4 \int_{\Omega_{\tau}}\left(\frac{1}{4}\left(V^{0}\right)^{2}+\left(V^{j}\right)^{2}\right) d x .
$$

Therefore we can choose $\varepsilon_{0}$ such that

$$
|u|<\varepsilon_{0} \Rightarrow \int_{F_{1}}>\frac{1}{8}\|V\|_{\Omega_{t}}^{2} .
$$

On $F_{3}$, we have $n_{0}=\gamma\left(1+\gamma^{2}\right)^{-1 / 2}, \sum n_{i}^{2}=\left(1+\gamma^{2}\right)^{-1}$. Hence:

$$
\left|n_{i} V^{0} V^{i}\right| \leqq \frac{1}{2 \alpha}\left(V^{0}\right)^{2}+\frac{\alpha}{2}\left(\sum n_{i}^{2}\right) \sum\left(V^{i}\right)^{2}, \text { for } \alpha>0 \text {. }
$$

Choosing $\alpha=2\left(1+\gamma^{2}\right)^{1 / 2}$, we have:

$$
\left|n_{i} V^{0} V^{i}\right| \leqq \frac{1}{4}\left(1+\gamma^{2}\right)^{-1 / 2}\left(V^{0}\right)^{2}+\left(1+\gamma^{2}\right)^{-1 / 2} \sum_{i}\left(V^{i}\right)^{2} .
$$

Since $\gamma>1$, there exists $C>0$ such that:

$$
n_{0}\left[\frac{1}{4}\left(V_{0}\right)^{2}+\sum_{i}\left(V^{i}\right)^{2}\right]+n_{i} V^{0} V^{i}>C|V|^{2} .
$$

Considering (II.B.3), we can choose $\varepsilon_{0}$ such that

$$
|u|<\varepsilon_{0} \Rightarrow\left|n_{i} k^{i}(u) V \otimes V\right|<C|V|^{2} .
$$

Then, we have $\int_{F_{3}}>0$.

Finally, considering the form (I.12) of the functions $f$ and $|\nabla u|<M_{0}$, we obtain

$$
\|V\|_{\Omega_{t}}^{2} \leqq C \int_{t_{0}}^{t}\left\{\operatorname{Sup}_{\Omega_{\tau}}|\nabla u|\|V\|_{\Omega_{\tau}}^{2}+\|V\|_{\Omega_{\tau}}\left(\|\phi\|_{\Omega_{\tau}}+\|\nabla \phi\|_{\Omega_{\tau}}\right)\right\} d \tau .
$$

Considering (II.A.5), Gronwall's lemma implies:

$$
\|V\|_{\Omega_{t}} \leqq C \int_{-\infty}^{t}\left(\|\phi\|_{\Omega_{\tau}}+\|\nabla \phi\|_{\Omega_{\tau}}\right) d \tau
$$

Remark. The same reasoning shows that, if $u$ verifies (II.A.5) the solutions $V$ of $L(u) V=g$, have the estimate

$$
\|V\|_{\Omega_{\mathrm{t}}} \leqq C \int_{-\infty}^{t}\|g\|_{\Omega_{\tau}} d \tau
$$


2. Estimates for the Derivatives of $V$. Deriving the Eqs. (II.B.1), we obtain, if $|\alpha|=i$ $(=1,2,3)$

$$
L\left(D^{\alpha} V\right)=f_{\alpha}
$$

with $f_{\alpha}^{A}=D^{\alpha} f^{A}+\sum_{k=1}^{i} \nabla^{k} u \otimes \nabla^{i+1-k} V$.

Proceeding as in 1), we obtain

$$
\left\|\nabla^{i} V\right\|_{\Omega_{t}}^{2} \leqq \int_{t_{0}}^{t} d \tau \int_{\Omega_{\tau}} \underbrace{}_{|\alpha|=i}\left(f_{\alpha}^{0} D^{\alpha} V^{0}+2 \sum_{j} f_{\alpha}^{j} D^{\alpha} V^{j}\right)+k(u) \nabla u \nabla^{i} V \otimes \nabla^{i} V\} d x .
$$

Considering (I.12), $H^{(1)}$ can be written as:

$$
\begin{aligned}
H^{(1)}= & k(u) \nabla u \otimes \nabla V \otimes \nabla V+\left[\left(k(u) \nabla u \otimes \nabla u+k(u) \nabla^{2} u\right) \otimes V\right. \\
& +\left(k(u) \otimes \nabla u \otimes \nabla u+k(u) \nabla^{2} u\right) \otimes \phi \\
& \left.+k(u) \nabla u \otimes \nabla \phi+k(u) \nabla^{2} \phi\right] \otimes \nabla V .
\end{aligned}
$$

Then, we have:

$$
\int_{\Omega_{\tau}} H^{(1)} d x \leqq C\left\{\operatorname{Sup}_{\Omega_{\tau}}|\nabla u|\|\nabla V\|_{\Omega_{\tau}}^{2}+\left\|\nabla V_{\Omega_{\tau}}\right\|\left(\|V\|_{\Omega_{\tau}}+\sum_{k=0}^{2}\left\|\nabla^{k} \phi\right\|_{\Omega_{\tau}}^{2}\right)\right\} .
$$

Gronwall's lemma leads to an estimate

$$
\|\nabla V\|_{\Omega_{t}} \leqq C \int_{-\infty}^{t}\left\{\|V\|_{\Omega_{\tau}}+\sum_{k=0}^{2}\left\|\nabla^{k} \phi\right\|_{\Omega_{\tau}}\right\} d \tau,
$$

and the estimate (II.B.4), leads to:

$$
\|\nabla V\|_{\Omega_{\tau}} \leqq C \int_{-\infty}^{t}\left\{(t-\tau+1) \sum_{k=0}^{1}\left\|\nabla^{k} \phi\right\|_{\Omega_{\tau}}+\left\|\nabla^{2} \phi\right\|_{\Omega_{\tau}}\right\} d \tau
$$

Estimates for $\left\|\nabla^{2} V\right\|_{\Omega_{t}}$ and $\left\|\nabla^{3} V\right\|_{\Omega_{t}}$ are obtained with the same process.

Some terms are tricky to estimate in $\int_{\Omega_{\tau}} H^{(2)} d x$ or in $\int_{\Omega_{\tau}} H^{(3)} d x$ :

$$
\begin{gathered}
\|\| \nabla^{3} u\|V\|\left\|_{\Omega_{\tau}} \leqq\right\| \nabla^{3} u\left\|_{L^{4}\left(\Omega_{\tau}\right)}\right\| V\left\|_{L^{4}\left(\Omega_{\tau}\right)} \leqq C \sum_{k=3}^{4}\right\| \nabla^{k} u\left\|_{\Omega_{\tau}} \sum_{k=0}^{1}\right\| \nabla^{k} V \|_{\Omega_{\tau}} \\
\leqq C^{\prime} \sum_{k=0}^{1}\left\|\nabla^{k} V\right\|_{\Omega_{\tau}}, \\
\|\| \nabla^{4} u|| V\left|\left\|_{\Omega_{\tau}} \leqq\right\| \nabla^{4} u\left\|_{\Omega_{\tau}} \operatorname{Sup}|V| \leqq C\right\| \nabla^{4} u\left\|_{\Omega_{\tau}} \sum_{k=0}^{2}\right\| \nabla^{k} V \|_{\Omega_{\tau}}\right. \\
\leqq C^{\prime} \sum_{k=0}^{2}\left\|\nabla^{k} V\right\|_{\Omega_{\tau}} .
\end{gathered}
$$

Finally, we obtain the estimates, for $i=0, \ldots, 3$

$$
\left\|\nabla^{i} V\right\|_{\Omega_{t}} \leqq C \int_{-\infty}^{t}\left\{(t-\tau+1)^{i}\|\phi\|_{\Omega_{\tau}}+\sum_{k=1}^{i+1}(t-\tau+1)^{i+1-k}\left\|\nabla^{k} \phi\right\|_{\Omega_{\tau}}\right\} d \tau \text {.(II.B.7) }
$$


3. Solutions. Starting from the results of Leray-Dionne $[8,9]$, with the estimates (II.B.7), we obtain

Lemma II.B. If $\phi$ verifies:

$$
\int_{-\infty}^{T}\left\{(T-t+1)^{3}\|\phi\|_{\Omega_{t}}+\sum_{k=1}^{4}(T-t+1)^{4-k}\left\|\nabla^{k} \phi\right\|_{\Omega_{t}}\right\} d t<+\infty,
$$

and if $u$ verifies (II.A.5, 7, 9), the system (II.B.1) has a unique solution $V \in E_{3}(\Omega)$. This solution verifies the estimates (II.B.7).

C. The Coupled System (I.10). In order to solve (I.10), we consider the system

$$
\left\{\begin{array}{l}
g^{\lambda \mu}(u) \partial_{\lambda \mu} u=-h(v) \nabla v \otimes \nabla v+l(v, V, \phi), \\
L(v) V=f(v, V, \phi)
\end{array}\right.
$$

In order to apply the results of parts II.A and II.B to (II.C.1) and (II.C.2), we must choose $v$ in a function-space such that $h(v) \nabla v \otimes \nabla v \in \mathscr{L}(\Omega)$.

We must also obtain $u$ in the same function space, where we choose $v$, in such a way that we can iterate and show that the application $v \mapsto u$ is contractant in some function space.

That leads us to introduce the norms $\tilde{q}_{\Omega}, \tilde{q}_{\Omega}^{*}$, and $p_{\Omega}^{1}$ (cf. $\S$ "function spaces and norms" at the beginning of part II). We shall choose $v$ in $\widetilde{E}^{*}(\Omega)$ and show that $v \mapsto u$ is a contractant for the norm $\tilde{q}_{\Omega}$.

Lemma II.C.1. If $p_{\Omega}(\varrho)<r$, and $u$ verifies $g^{\alpha \beta}(u) \partial_{\alpha \beta} u=\varrho$, then

$$
\tilde{q}_{\Omega}^{*}(u)<C p_{\Omega}(\varrho)<C r .
$$

If $\varrho^{1}$ and $\varrho^{2}$ verify (II.C.3), then

$$
\tilde{q}_{\Omega}\left(u_{1}-u_{2}\right) \leqq C p_{\Omega}^{1}\left(\varrho^{1}-\varrho^{2}\right) .
$$

Proof of (II.C.4). Considering (II.A.8), it is sufficient to show that

Now, we have:

$$
\int_{-\infty}^{T}(T-t+1) \operatorname{Sup}_{\tau \leqq t}\left(\sum_{i=0}^{3}\left\|\nabla^{i} u\right\|_{\Omega_{\tau}}\right) d t<C p_{\Omega}(\varrho)
$$

$$
\operatorname{Sup}_{\tau \leqq t}\left(\sum_{i=0}^{3}\left\|\nabla^{i} u\right\|_{\Omega_{\tau}}\right) \leqq C \int_{-\infty}^{t}\left\{(t-\tau+1)\left(\|\varrho\|_{\Omega_{\tau}}+\|\nabla \varrho\|_{\Omega_{\tau}}\right)+\left\|\nabla^{2} \varrho\right\|_{\Omega_{\tau}}\right\} d \tau
$$

Hence:

$$
\begin{aligned}
\int_{-\infty}^{T}(T-t+1) \operatorname{Sup}_{\tau<t}\left(\sum_{i=0}^{3}\left\|\nabla^{i} u\right\|_{\Omega_{\tau}}\right) d t \leqq & C \int_{-\infty}^{T}\left\{(T-t+1)^{3}\left(\|\varrho\|_{\Omega_{t}}+\|\nabla \varrho\|_{\Omega_{t}}\right)\right. \\
& \left.+(T-t+1)^{2}\left\|\nabla^{2} \varrho\right\|_{\Omega_{t}}\right\} d t . \quad \text { q.e.d. }
\end{aligned}
$$

Proof of (II.C.5). $u_{1}-u_{2}$ verifies the system

where $\tilde{\varrho}=\tilde{\varrho}^{\prime}+\tilde{\varrho}^{\prime \prime}$, with

$$
g^{\alpha \beta}\left(u_{1}\right) \partial_{\alpha \beta}\left(u_{1}-u_{2}\right)=\tilde{\varrho},
$$

$$
\left\{\begin{array}{l}
\tilde{\varrho}^{\prime}=\varrho_{1}-\varrho_{2}, \\
\tilde{\varrho}^{\prime \prime}=\left(g^{\alpha \beta}\left(u_{2}\right)-g^{\alpha \beta}\left(u_{1}\right)\right) \partial_{\alpha \beta} u_{2},
\end{array}\right.
$$


and we can follow the same kind of reasoning as in part II.A, Theorem 2; so we find:

$$
\sum_{k=0}^{3}\left\|\nabla^{k}\left(u_{1}-u_{2}\right)\right\|_{\Omega_{t}} \leqq C \int_{-\infty}^{t}\left\{(t-\tau) \sum_{k=0}^{1}\left\|\nabla^{k}\left(\varrho_{1}-\varrho_{2}\right)\right\|_{\Omega_{\tau}}+\left\|\nabla^{2}\left(\varrho_{1}-\varrho_{2}\right)\right\|_{\Omega_{\tau}}\right\} d \tau .
$$

Then we have:

$$
\begin{aligned}
\int_{-\infty}^{T} & (T-t+1) \operatorname{Sup}_{\tau \leqq t}\left(\sum_{k=0}^{3}\left\|\nabla^{k}\left(u_{1}-u_{2}\right)\right\|_{\Omega_{\tau}}\right) d t \\
\leqq & \int_{-\infty}^{T}\left\{(T-t+1)^{3} \sum_{k=0}^{1}\left\|\nabla^{k}\left(\varrho_{1}-\varrho_{2}\right)\right\|_{\Omega_{t}}\right. \\
& \left.+(T-t+1)^{2}\left\|\nabla^{2}\left(\varrho_{1}-\varrho_{2}\right)\right\|_{\Omega_{t}}\right\} d t .
\end{aligned}
$$

Inequalities (II.C.6) and (II.C.7) imply (II.C.5).

Lemma II.C.2. $\forall r_{0}>0$, there exists $C_{1}\left(r_{0}\right)>0$, such that $C_{1}\left(r_{0}\right) \rightarrow 0$ when $r_{0} \rightarrow 0$, and

$$
\tilde{q}_{\Omega}^{*}(v)<r_{0} \Rightarrow p_{\Omega}(h(v) \nabla v \otimes \nabla v)<C_{1}\left(r_{0}\right) \tilde{q}_{\Omega}(v),
$$

$\forall r_{0}>0$, there exists $C_{2}\left(r_{0}\right)>0$, such that $C_{2}\left(r_{0}\right) \rightarrow 0$ when $r_{0} \rightarrow 0$, and

$$
\begin{aligned}
& \tilde{q}_{\Omega}^{*}\left(v_{1}\right)<r_{0} \quad \text { and } \quad \tilde{q}_{\Omega}^{*}\left(v_{2}\right)<r_{0} \Rightarrow p_{\Omega}^{1}\left(h\left(v_{1}\right) \nabla v_{1} \otimes \nabla v_{1}-h\left(v_{2}\right) \nabla v_{2} \otimes \nabla v_{2}\right) \\
& \quad \leqq C_{2}\left(r_{0}\right) \tilde{q}_{\Omega}\left(v_{1}-v_{2}\right) \text {. }
\end{aligned}
$$

Proof of (II.C.8). We show on some terms of $p_{\Omega}$, how the estimates are done. For instance, there is the term

$$
\int_{-\infty}^{T}(T-t+1)^{3}\|\nabla(h(v) \nabla v \otimes \nabla v)\|_{\Omega_{t}} d t
$$

Since

$$
\nabla(h(v) \nabla v \otimes \nabla v)=k(v) \nabla v^{3}+k(v) \nabla v \otimes \nabla^{2} v,
$$

(we write $\nabla v^{3}=\nabla v \otimes \nabla v \otimes \nabla v$ ), we must estimate:

$$
\begin{gathered}
A=\int_{-\infty}^{T}(T-t+1)^{3}\left\|k(v) \nabla v \otimes \nabla^{2} v\right\|_{\Omega_{\tau}} d \tau \leqq C \int_{-\infty}^{T}(T-\tau+1)^{3} \operatorname{Sup}_{\Omega_{\tau}}|\nabla v|\left\|\nabla^{2} v\right\|_{\Omega_{\tau}} d \tau \\
A \leqq C \int_{-\infty}^{T}(T-\tau+1) \operatorname{Sup}_{\Omega_{\tau}}|\nabla v|\left\|\nabla^{2} v\right\|_{\Omega_{\tau}} d \tau\left(\int_{\tau}^{T}(T-t+1) d t+1\right) \\
A \leqq C \int_{-\infty}^{T}(T-t+1) d t \int_{-\infty}^{t}(T-\tau+1) \underset{\Omega_{\tau}}{\operatorname{Sup}}|\nabla v|\left\|\nabla^{2} v\right\|_{\Omega_{\tau}} d \tau \\
+C \int_{-\infty}^{T}(T-\tau+1) \operatorname{Sup}_{\Omega_{\tau}}|\nabla v|\left\|\nabla^{2} v\right\|_{\Omega_{\tau}} d \tau \\
A \leqq C \int_{-\infty}^{T}(T-t+1) \operatorname{Sup}_{\tau<t} \operatorname{Sup}_{\Omega_{\tau}}|\nabla v| d t \int_{-\infty}^{T}(T-\tau+1)\left\|\nabla^{2} v\right\|_{\Omega_{\tau}} d \tau \\
+\operatorname{Sup}_{\tau \leqq t}|\nabla v| \int_{-\infty}^{T}(T-t+1)\left\|\nabla^{2} v\right\|_{\Omega_{\tau}} d \tau
\end{gathered}
$$


Hence, considering Sobolev's estimates, $A \leqq \tilde{q}_{\Omega}(v) \tilde{q}_{\Omega}(v)$. Another instance: there is the term:

$$
\begin{gathered}
\int_{-\infty}^{T}(T-t+1)\left\|\nabla^{3}(h(v) \nabla v \otimes \nabla v)\right\|_{\Omega_{t}} d t \\
\nabla^{3}(h(v) \nabla v \otimes \nabla v)= \\
k(v) \nabla v \otimes \nabla^{4} v+k(v) \nabla^{2} v \otimes \nabla^{3} v+k(v) \nabla v^{2} \otimes \nabla^{3} v \\
+k(v) \nabla v \otimes \nabla^{2} v \otimes \nabla^{2} v+k(v) \nabla v^{3} \otimes \nabla^{2} v+k(v) \nabla v^{5}
\end{gathered}
$$

We must estimate for instance

$$
\begin{aligned}
& A=\int_{-\infty}^{T}(T-\tau+1)\left\|k(v) \nabla v \otimes \nabla^{4} v\right\|_{\Omega_{\tau}} d \tau \leqq C \int_{-\infty}^{T}(T-\tau+1) \operatorname{Sup}_{\Omega_{\tau}}|\nabla v|\left\|\nabla^{4} v\right\|_{\Omega_{\tau}} d \tau, \\
& A \leqq C \underset{\tau<T}{\operatorname{Ess}} \operatorname{Sup}\left\|\nabla^{4} v\right\|_{\Omega_{\tau}} \int_{-\infty}^{T}(T-\tau+1) \sum_{k=1}^{3}\left\|\nabla^{k} v\right\|_{\Omega_{\tau}} d \tau \leqq C \tilde{q}_{\Omega}^{*}(v) \tilde{q}_{\Omega}(v) .
\end{aligned}
$$

Proof of (II.C.9). We can write

$$
\begin{aligned}
h\left(v_{1}\right) \nabla v_{1} \otimes \nabla v_{1}-h\left(v_{2}\right) \nabla v_{2} \otimes & \nabla v_{2} \\
=h\left(v_{1}\right)\left(\nabla\left(v_{2}-v_{1}\right) \otimes \nabla v_{1}\right. & \left.+\nabla v_{2} \otimes \nabla\left(v_{2}-v_{1}\right)\right) \\
& +k\left(v_{1}, v_{2}\right)\left(v_{2}-v_{1}\right) \otimes \nabla v_{2} \otimes \nabla v_{2} .
\end{aligned}
$$

Since, $h$ being an analytic function, we can write

$$
h\left(v_{1}\right)-h\left(v_{2}\right)=k\left(v_{1}, v_{2}\right)\left(v_{2}-v_{1}\right),
$$

where $k\left(v_{1}, v_{2}\right)$ is an analytic function of $v_{1}$ and $v_{2}$ in the neighbourhood of $(0,0)$. The continuation of the proof is through estimates similar to the proof of II.C.8.

Lemma II.C.3. 1. If $v$ verifies (II.A.5, 7, 9) and if $L(v) V=f(v, V, \phi)$, if $\hat{p}_{\Omega}(\phi)<+\infty$, then,

$$
p_{\Omega}(V)<C \hat{p}_{\Omega}(\phi) .
$$

2. If $v_{1}$ and $v_{2}$ verify (II.A.5, 7, 9), if $\hat{p}_{\Omega}(\phi)<+\infty$, and if

$$
\begin{aligned}
& L\left(v_{1}\right) V_{1}=f\left(v_{1}, V_{1}, \phi\right), \\
& L\left(v_{2}\right) V_{2}=f\left(v_{2}, V_{2}, \phi\right),
\end{aligned}
$$

then

$$
p_{\Omega}^{1}\left(V_{1}-V_{2}\right) \leqq C \hat{p}_{\Omega}(\phi) \tilde{q}_{\Omega}\left(v_{1}-v_{2}\right)
$$

Proof of (II.C.10). It is a consequence of Lemma II.B and estimates II.B.7.

Proof of (II.C.11). $V_{1}-V_{2}$ verifies the system $L\left(v_{1}\right)\left(V_{1}-V_{2}\right)=g$, where $g=k\left(v_{1}\right) \nabla v_{1}\left(V_{1}-V_{2}\right)+g^{\prime}$,

$$
\begin{aligned}
g^{\prime}= & \left(k\left(v_{1}\right) \nabla\left(v_{1}-v_{2}\right)+k\left(v_{1}, v_{2}\right)\left(v_{1}-v_{2}\right) \otimes \nabla v_{2}\right) \otimes V_{2} \\
& +\left(k\left(v_{1}\right) \nabla\left(v_{1}-v_{2}\right)+k\left(v_{1}, v_{2}\right)\left(v_{1}-v_{2}\right) \otimes \nabla v_{2}\right) \otimes \phi \\
& +k\left(v_{1}, v_{2}\right)\left(v_{1}-v_{2}\right) \otimes \nabla \phi .
\end{aligned}
$$


Following the same process as in part II.B, we find

therefore

$$
\left\|V_{1}-V_{2}\right\|_{\Omega_{\tau}} \leqq C \int_{-\infty}^{t}\left\|g^{\prime}\right\|_{\Omega_{\tau}} d \tau
$$

$$
\left\|V_{1}-V_{2}\right\|_{\Omega_{\tau}} \leqq C \operatorname{Sup}_{\tau \leqq t}\left(\sum_{i=0}^{3}\left\|\nabla^{i}\left(v_{1}-v_{2}\right)\right\|_{\Omega_{\tau}}\right) \int_{-\infty}^{t}\left\{\left\|V_{2}\right\|_{\Omega_{\tau}}+\|\phi\|_{\Omega_{t}}+\|\nabla \phi\|_{\Omega_{\tau}}\right\} d \tau,
$$

and considering (II.B.7) verified by $V_{2}$,

$$
\left\|V_{1}-V_{2}\right\|_{\Omega_{t}} \leqq C \operatorname{Sup}_{\tau \leqq t}\left(\sum_{i=0}^{3}\left\|\nabla^{i}\left(v_{1}-v_{2}\right)\right\|_{\Omega_{\tau}}\right) \int_{-\infty}^{t}(t-\tau+1)\left(\|\phi\|_{\Omega_{\tau}}+\|\nabla \phi\|_{\Omega_{\tau}}\right) d \tau .
$$

Similarly starting from the derivated equations, we find, for $i=0,1,2$,

$$
\begin{aligned}
\left\|\nabla^{i}\left(V_{1}-V_{2}\right)\right\|_{\Omega_{t}} \leqq & C \operatorname{Sup}_{\tau \leqq t}\left(\sum_{i=0}^{3}\left\|\nabla^{i}\left(v_{1}-v_{2}\right)\right\|_{\Omega_{\tau}}\right) \int_{-\infty}^{t}\left\{(t-\tau+1)^{i+1}\|\phi\|_{\Omega_{\tau}}\right. \\
& \left.+\sum_{k=1}^{i+1}(t-\tau+1)^{i+2-k}\left\|\nabla^{k} \phi\right\|_{\Omega_{\tau}}\right\} d \tau
\end{aligned}
$$

From (II.C.12), we can deduce (II.C.11).

For instance in $p_{\Omega}^{1}\left(V_{1}-V_{2}\right)$, we must estimate:

$$
\begin{gathered}
A=\int_{-\infty}^{T}(T-t+1)^{2}\left\|\nabla^{2}\left(V_{1}-V_{2}\right)\right\|_{\Omega_{\tau}} d t \\
\leqq \\
\qquad \int_{-\infty}^{t} d t(T-\tau+1)^{2} \operatorname{Sup}_{\tau \leqq t}\left(\sum_{i=0}^{3}\left\|\nabla^{i}\left(v_{1}-v_{2}\right)\right\|_{\Omega_{\tau}}\right) \\
\quad \times \int_{-\infty}^{t}\left\{(t-\tau+1)^{3}\|\phi\|_{\Omega_{\tau}}+\sum_{k=1}^{3}(t-\tau+1)^{4-k}\left\|\nabla^{k} \phi\right\|_{\Omega_{\tau}}\right\} d \tau, \\
A \leqq C \sum_{k=1}^{3} \int_{-\infty}^{T}\left\|\nabla^{k} \phi\right\|_{\Omega_{\tau}} d \tau \int_{\tau}^{T}(T-t+1)^{2}(t-\tau+1)^{4-k} \operatorname{Sup}_{\tau \leqq t}\left(\sum_{i=0}^{3}\left\|\nabla^{i}\left(v_{1}-v_{2}\right)\right\|_{\Omega_{\tau}}\right) d t \\
+C \int_{-\infty}^{T}\|\phi\|_{\Omega_{\tau}} d \tau \int_{\tau}^{T}(T-t+1)^{2}(t-\tau+1)^{3} \operatorname{Sup}_{\tau \leqq t}\left(\sum_{i=0}^{3}\left\|\nabla^{i}\left(v_{1}-v_{2}\right)\right\|_{\Omega_{\tau}}\right) d t, \\
A \leqq C \sum_{k=1}^{3} \int_{-\infty}^{T}(T-\tau+1)^{5-k}\left\|\nabla^{k} \phi\right\|_{\Omega_{\tau}} d \tau \int_{-\infty}^{T}(T-t+1) \operatorname{Sup}_{\tau \leqq t}\left(\sum_{i=0}^{3}\left\|\nabla^{i}\left(v_{1}-v_{2}\right)\right\|_{\Omega_{\tau}}\right) d t \\
+C \int_{-\infty}^{T}(T-\tau+1)^{4}\|\phi\|_{\Omega_{\tau}} d \tau \int_{-\infty}^{T}(T-t+1) \underset{\tau \leqq t}{\operatorname{Sup}}\left(\sum_{i=0}^{3}\left\|\nabla^{i}\left(v_{1}-v_{2}\right)\right\|_{\Omega_{\tau}}\right) d t, \\
A \leqq C \hat{p}_{\Omega}(\phi) \tilde{q}_{\Omega}\left(v_{1}-v_{2}\right) .
\end{gathered}
$$

Lemma II.C.4. There exist constants $r_{0}$ and $\hat{r}$ such that if $\hat{p}_{\Omega}(\phi)<\hat{r}$,

1. if $\hat{q}_{\Omega}^{*}(v)<r_{0}$, the system (II.C.1) (II.C.2), has a unique solution $(u, V)$ such that $\tilde{q}_{\Omega}^{*}(u)<r_{0}$ and $V \in E_{3}(\Omega)$.

2. The application $v \mapsto u$ so defined in $F=\left\{v \in \widetilde{E}^{*}(\Omega), \tilde{q}_{\Omega}^{*}(v)<r_{0}\right\}$ is contractant in $F$ with the topology induced by $\widetilde{E}(\Omega)$. 
Proof of 1). We set $\varrho=-h(v) \nabla v \otimes \nabla v+l(v, V, \phi)$.

From Lemmas II.C.2 and II.C.3, we can deduce, if $\tilde{q}_{\Omega}^{*}(v)<r_{0}$,

$$
p_{\Omega}(\varrho) \leqq C_{1}\left(r_{0}\right) \tilde{q}_{\Omega}(v)+C \tilde{p}_{\Omega}(\phi) .
$$

Then we can determine $r_{0}^{\prime}$ and $\hat{r}_{1}$ such that

$$
\tilde{q}_{\Omega}^{*}(v)<r_{0}^{\prime} \quad \text { and } \quad \hat{p}_{\Omega}(\phi)<\hat{r}_{1} \Rightarrow p_{\Omega}(\varrho)<r .
$$

Then we can apply to (II.C.1) Theorem 2 and Lemma II.C.1. (II.C.1) has a unique solution $u$ such that

$$
\tilde{q}_{\Omega}^{*}(u)<K p_{\Omega}(\varrho) \leqq K\left[C_{1}\left(r_{0}\right) \tilde{q}_{\Omega}(v)+C \hat{p}_{\Omega}(\phi)\right] .
$$

Then we determine $r_{0}^{\prime \prime} \leqq r_{0}^{\prime}$ and $\alpha>0$ such that

$$
\tilde{q}_{\Omega}^{*}(v)<r_{0}^{\prime \prime} \quad \text { and } \quad \hat{p}_{\Omega}(\phi)<\alpha r_{0}^{\prime \prime}<\hat{r}_{1} \Rightarrow \tilde{q}_{\Omega}^{*}(u)<r_{0}^{\prime \prime},
$$

using the fact that $C_{1}\left(r_{0}\right) \rightarrow 0$, when $r_{0} \rightarrow 0$. We set $\hat{r}_{2}=\alpha r_{0}^{\prime \prime}$.

Proof of 2). From (II.C.5), (II.C.9), and (II.C.11), we can deduce an estimate:

$$
\tilde{q}_{\Omega}\left(u_{1}-u_{2}\right) \leqq \tilde{q}_{\Omega}\left(v_{1}-v_{2}\right)\left[K C_{2}\left(r_{0}\right)+K C \hat{p}_{\Omega}(\phi)\right] .
$$

Using the fact that $C_{2}\left(r_{0}\right) \rightarrow 0$ when $r_{0} \rightarrow 0$, we can choose $r_{0}<r_{0}^{\prime \prime}$ and $\hat{r} \leqq \hat{r}_{2}$, such that $K C_{2}\left(r_{0}\right)+K C \hat{r}<\frac{1}{2}$. q.e.d.

Lemma II.C.5. $F=\left\{v \in \widetilde{E}^{*}(\Omega), \tilde{q}_{\Omega}^{*}(v) \leqq r_{0}\right\}$, with the topology induced by $\widetilde{E}(\Omega)$, is a complete metric space.

Proof. We use the weak compactness of the balls of

$$
Q_{0}(\Omega)=\left\{f \text { measurable on } \Omega \mid\|f\|_{Q_{0}}=\underset{t<T}{\operatorname{Ess} \operatorname{Sup}}\|f\|_{\Omega_{t}}<+\infty\right\} .
$$

If $v_{n}$ is a Cauchy sequence in $F$, for the metric,

$$
d\left(v_{p}, v_{q}\right)=\tilde{q}_{\Omega}\left(v_{p}-v_{q}\right), \quad v_{p} \text { converges to } v \text { in } \tilde{E}(\Omega) .
$$

But we can find a subsequence, $v_{p}$ such that $\nabla^{4} v_{p}$ converges weakly in $Q_{0}(\Omega)$, to $w$. Then we have $\nabla^{4} v=w \in Q_{0}(\Omega)$ and $\tilde{q}_{\Omega}^{*}(v) \leqq r_{0}$.

Lemmas II.C.4 and II.C.5 imply the first part of the main theorem.

D. Gauge Conditions. If Eqs. (I.1) and (I.2) are verified, the $\Phi^{\lambda}$ of (I.3) verify a system of equations (cf. [10])

$$
g^{\alpha \beta} \partial_{\alpha \beta} \Phi^{\lambda}+k(g) \nabla g \otimes \nabla \Phi=0 .
$$

$\Phi^{\lambda}$ has the form $\Phi^{\lambda}=k(u) \nabla u$ and we can apply to the system (II.D.1) the results of part II.A (Proposition 3), then

Hence

$$
\|\nabla \Phi\|_{\Omega_{t}} \leqq K_{0} \int_{-\infty}^{t}\|k(u) \nabla u \otimes \nabla \Phi\|_{\Omega_{\tau}} d \tau
$$

$$
\|\nabla \Phi\|_{\Omega_{t}} \leqq C \int_{-\infty}^{t} \operatorname{Sup}|\nabla u|\|\nabla \Phi\|_{\Omega_{\tau}} d \tau
$$

and Gronwall's lemma imply $\|\nabla \Phi\|_{\Omega_{t}}=0$, that is $\phi=0$. 


\section{Positivity Conditions for the Solutions}

Do the solutions we have determined on $V^{T}$ verify the weak positivity condition? That is,

$$
T^{\alpha \beta} X_{\alpha} X_{\beta} \geqq 0 \quad \text { for all timelike vector fields } X_{\alpha} .
$$

The $T^{\alpha \beta}$ are determined in terms of the data $\phi^{i j}$ and of the solution $\left(g_{\alpha \beta}, V^{A}\right)$, through

$$
T^{A B}=\varrho^{A B}-\frac{1}{2} \eta^{A B} \eta_{C D} \varrho^{C D}, \text { where } \varrho^{0 A}=V^{A}, \varrho^{I J}=\phi^{I J} .
$$

Since as $t \rightarrow-\infty, g_{\alpha \beta} \rightarrow \eta_{\alpha \beta}$ or $e_{A}^{\alpha} \rightarrow \delta_{A}^{\alpha}$, the conservation equations

are approximately

$$
\left|\nabla_{A} T^{A B}=\right| \nabla_{A}\left(\varrho^{A B}-\frac{1}{2} \eta^{A B} \eta_{C D} \varrho^{C D}\right)=0
$$

and (III.2) can be written

$$
\partial_{\alpha}\left(\varrho^{\alpha \beta}-\frac{1}{2} \eta^{\alpha \beta}\left(\varrho^{00}-\sum_{i} \varrho^{i i}\right)\right)=0
$$

a)

$$
\left\{\begin{array}{l}
\frac{1}{2} \partial_{0} V^{0}+\partial_{i} V^{i}=-\frac{1}{2} \partial_{0}\left(\sum_{i} \phi^{i i}\right), \\
\frac{1}{2} \partial_{j} \mathrm{~V}^{0}+\partial_{0} \mathrm{~V}^{j}=\frac{1}{2} \partial_{j}\left(\sum_{i} \phi^{i i}\right)-\sum_{i} \partial_{i} \phi^{i j},
\end{array} \quad i, j=1, \ldots, 3 .\right.
$$

It is reasonable to think that, near $-\infty$, the $V^{A}$ of the solution of (I.10) determined by the data $\phi^{i j}$ are close to the $V^{A}$ solution of (III.3) for the same data.

We call the system (III.3) "approximated equations", and we shall first seek whether some $\phi^{i j}$ can be found such that the approximated equations have a solution $V^{A}$ verifying the positivity condition for the Minkowski metric. After that, with the $\phi^{i j}$ so determined, we shall seek whether the exact solution, that is the solution of (I.10), verifies the positivity condition.

\section{A. Approximated Equations}

1. Explicit Solution of the Approximated Equations. Through derivation of (III.3a) by $\partial_{0}$ and (III.3b) by $\partial_{j}$ we obtain:

$$
\begin{aligned}
\frac{1}{2}\left(\partial_{00}-\sum_{i} \partial_{i i}\right) V^{0}= & -\frac{1}{2}\left(\partial_{00}+\sum_{j} \partial_{j j}\right)\left(\sum_{i} \phi^{i i}\right)+\sum_{i, j} \partial_{i j} \phi^{i j} .
\end{aligned}
$$

The fact that $V^{0} \rightarrow 0$ when $t \rightarrow-\infty$, shows that (III.4) has a unique solution, given by Kirchoff's formula:

$$
V^{0}(t, x)=\int_{R^{3}} \frac{1}{4 \pi|\xi|} 2 h(t-|\xi|, x-\xi) d \xi
$$

Equations (III.3b) can be written:

$$
\partial_{0} V^{j}=-\frac{1}{2} \partial_{j} V^{0}+\frac{1}{2} \partial_{j}\left(\sum_{i} \phi^{i i}\right)-\sum_{i} \partial_{i} \phi^{i j}
$$

The fact that $V^{j} \rightarrow 0$ when $t \rightarrow-\infty$ shows that $V^{j}$ is uniquely determined by:

$$
V^{j}(x, t)=\int_{-\infty}^{t} g_{j}(\tau, x) d \tau
$$


Since the positivity condition is expressed through the $T^{A B}$, linked with the $V^{A}$ and the $\phi^{I J}$ by:

$$
\begin{aligned}
T^{00} & =\frac{1}{2}\left(V^{0}+\sum_{i} \phi^{i i}\right) \\
T^{0 i} & =V^{i} \\
T^{11} & =\frac{1}{2}\left(V^{0}+\phi^{11}-\phi^{22}-\phi^{33}\right), \text { and similar formulas for } T^{22}, T^{33}, \\
T^{i j} & =\phi^{i j} \quad \text { if } i \neq j
\end{aligned}
$$

we shall express the $T^{A B}$ in terms of the $\phi^{I J}$,

$$
\begin{aligned}
& \begin{aligned}
& T^{00}(t, x)= \int_{R^{3}} \frac{1}{4 \pi|\xi|} f(t-|\xi|, x-\xi) d \xi \\
& \text { with } \begin{aligned}
f= & -\left(\partial_{22}+\partial_{33}\right) \phi^{11}-\left(\partial_{33}+\partial_{11}\right) \phi^{22}-\left(\partial_{11}+\partial_{22}\right) \phi^{33} \\
& +2 \partial_{12} \phi^{12}+2 \partial_{23} \phi^{23}+2 \partial_{31} \phi^{31}
\end{aligned} \\
& \begin{aligned}
T^{0 j}(t, x)= & \int_{-\infty}^{t} g_{j}(\tau, x) d \tau
\end{aligned} \\
& \text { with } g_{j}=-\partial_{j} T^{00}+\partial_{j}\left(\sum_{i} \phi^{i i}\right)-\sum_{i} \partial_{i} \phi^{i j} .
\end{aligned} \\
& \begin{aligned}
T^{11}=T^{00}-\phi^{22}-\phi^{33}, \quad \text { and similar formulas for } T^{22} \text { and } T^{33} . \\
T^{i j}=\phi^{i j} \quad \text { if } i \neq j .
\end{aligned}
\end{aligned}
$$

Evidently $T^{00}, T^{0 j}$ are defined only if the integrals in the right-hand sides are convergent. But if the $\phi^{I J}$ verify $\hat{p}_{\Omega}(\phi)<\hat{r}, \forall \Omega$, these integrals are convergent and define locally integrable $T^{00}$, and $T^{0 j}$.

(We put

$$
F(x)=\int_{-\infty}^{T} d \tau \int_{R^{3}} \frac{1}{4 \pi|\xi|} f(\tau-|\xi|, x-\xi) d \xi .
$$

Through changes of variables, we obtain

\section{Then}

$$
F(x)=\int_{-\infty}^{T} d t \int_{|\xi|<T-t} \frac{1}{4 \pi|\xi|} f(t, x-\xi) d \xi .
$$

$$
\begin{aligned}
&\left|\int_{|\xi|<T-t} \frac{1}{4 \pi|\xi|} f(t, x-\xi) d \xi\right| \leqq \frac{1}{4 \pi}\left(\int_{B(x, T-t)} \frac{d \eta}{|x-\eta|^{2}}\right)^{1 / 2}\left(\int_{B(x, T-t)}|f(t, \eta)|^{2} d \eta\right)^{1 / 2} \\
& \leqq \frac{1}{\sqrt{4 \pi}}|T-t|^{1 / 2}\|f\|_{\Omega_{t}(x)} . \\
& \text { Hence } F(x) \leqq \int_{-\infty}^{T} \frac{1}{\sqrt{4 \pi}}|T-t|^{1 / 2}\|f\|_{\Omega_{t}(x)} d t
\end{aligned}
$$

Now $\hat{p}_{\Omega}(\phi)<\hat{r} \Rightarrow \int_{-\infty}^{T}(T-t+1)^{4}\|f\|_{\Omega_{\tau}} d t<\hat{r}: F(x)$ is then defined and also $T^{00}(t, x)$. The proof is similar for $\left.T^{0 j}(t, x)\right)$. 
2. Instance of $\phi^{I J}$ on $V^{T}$ Leading to $T^{A B}$ verifying the Positivity Condition. In order to simplify, we choose $T=-1$; so we have $T-t+1=|t|$ and $V^{T}=V^{-1}=\bigcup_{a \in R^{3}} \Omega(-1, a)$.

Also in order to simplify the solutions, we search $\phi^{I J}$ with the form:

$$
\left\{\begin{array}{l}
\phi^{12}=\phi^{23}=\phi^{31}=0 \\
\phi^{11}=\phi^{22}=\phi^{33}=\phi
\end{array}\right.
$$

where $\phi$ is a function of the two variables $t$ and $r=|x|$.

With such a choice of $\phi^{I J}$, the function $f$ in (III.6) is:

$$
f=-2 \Delta \phi \quad\left(\Delta=\partial_{11}+\partial_{22}+\partial_{33}\right) .
$$

In order to get $T^{00} \geqq 0$, which is necessary for the positivity condition, we choose $f \geqq 0$. When the function $f(t, r)$ is chosen, (III.7) shows that $\phi$ is determined by:

Hence

$$
\frac{\partial^{2} \phi}{\partial r^{2}}+\frac{2}{r} \frac{\partial \phi}{\partial r}=-\frac{1}{2} f
$$

$$
\begin{aligned}
& \frac{\partial \phi}{\partial r}=\frac{1}{r^{2}} \int_{0}^{r}-\frac{1}{2} f(t, \varrho) \varrho^{2} J \varrho, \\
& \phi(t, r)=\int_{0}^{r} \frac{\partial \phi}{\partial r}(t, \varrho) d \varrho+C(t),
\end{aligned}
$$

where $C(t)$ is an arbitrary function of $t$.

From the Kirchhoff's formula (cf. III.6) we see that $T^{00}(t, r)$ is determined by:

$$
T^{00}(t, r)=\frac{1}{2 r} \int_{D(t, r)} f(\tau, \varrho) \varrho d \tau d \varrho,
$$

where $D(t, r)$ is the domain of the $(\tau, \varrho)$-plane defined by

$$
\left(\begin{array}{l}
\tau \leqq t \\
\text { and }
\end{array}|t-\tau-r| \leqq \varrho \leqq t-\tau+r\right.
$$

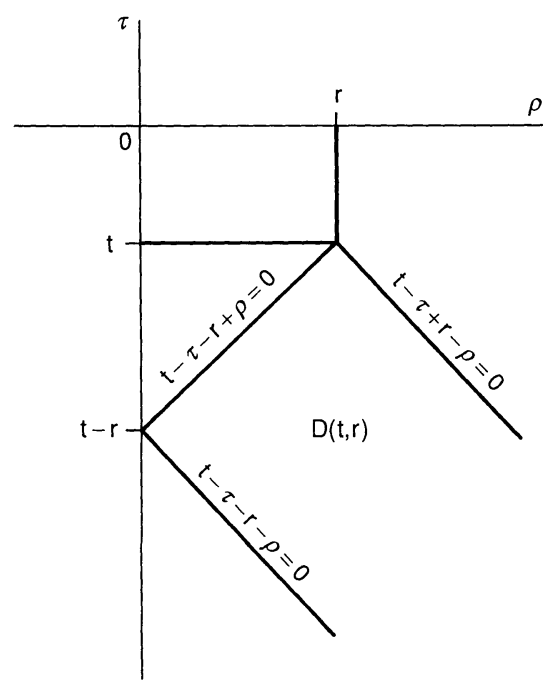


The simplest choice for $f$ is

$$
f=\frac{\delta_{0}}{|t|^{m}}, \quad \text { where } \delta_{0} \text { and } m \text { are positive constants }
$$

With such a choice, we have

$$
\phi(t, r)=C(t)-\frac{\delta_{0}}{12|t|^{m}} r^{2}
$$

Hence, $\phi(t, r)$ is not a bounded function as $r \rightarrow+\infty$. This is incompatible with the condition $\hat{p}_{\Omega}(\phi)<\hat{r}$ for all domains $\Omega(-1, a)$ since, with a domain $\Omega(-1, a)$ such that the point $a$ tends to infinity we have $\hat{p}_{\Omega}(\phi) \rightarrow+\infty$.

Then we may choose:

$$
f(t, r)= \begin{cases}\delta(t) \quad \text { if } \quad 0 \leqq r \leqq A(t), & \alpha>0, \\ \frac{\delta^{\prime}(t)}{r^{\alpha}} & \text { if } \quad r \geqq A(t),\end{cases}
$$

where $\delta, \delta^{\prime}, A$ are given positive functions of $t$.

Remark. The discontinuity of $f$ defined by (III.9) and of his derivatives for $r=A(t)$, is not compatible with the hypotheses of smoothness made on the $\phi^{I J}$. But, such a $f$ can be approached by smooth functions, e.g.:

$$
f_{\varepsilon}(t, r)=\left\{\begin{array}{l}
\frac{\delta^{\prime}}{r^{\alpha}} e^{-\frac{\varepsilon}{r^{2}}}+\left(\delta-\frac{\delta^{\prime}}{r^{\alpha}} e^{-\frac{\varepsilon}{r^{2}}}\right) e^{-\frac{\varepsilon}{A^{2}-r^{2}}} \text { if } \quad r<A(t) \\
\delta^{\prime} e^{-\frac{\varepsilon}{r^{2}}}, \quad \text { if } \quad r \geqq A(t) \\
r^{\alpha}
\end{array}\right.
$$

In order to verify positivity conditions, it is easier to use the $f$ defined by (III.9). The results will be true for the $f_{\varepsilon}$ for small enough $\varepsilon$. With $f$ defined by (III.9), we find (if $\alpha \neq 2$ and $\alpha \neq 3$ );

$$
\phi(t, r)=\left\{\begin{array}{l}
C(t)-\frac{\delta}{12} r^{2} \quad \text { if } \quad r \leqq A(t), \\
C(t)-\frac{\delta}{4} A^{2}-\frac{\delta^{\prime} A^{2-\alpha}}{2(\alpha-2)}+\left(\frac{\delta}{6} A^{3}+\frac{\delta^{\prime} A^{3-\alpha}}{2(\alpha-3)}\right) \frac{1}{r} \\
-\frac{\delta^{\prime}}{2(\alpha-3)(\alpha-2)} \cdot \frac{1}{r^{\alpha-2}} \quad \text { if } \quad r \geqq A(t),
\end{array}\right.
$$

where $C(t)$ is an arbitrary function of $t$.

In order to that $\phi$ be bounded as $r \rightarrow+\infty$, we must choose $\alpha>2$. $\delta$ and $\delta^{\prime}$ can be chosen as $\delta(t)=\frac{\delta_{0}}{|t|^{m}}, \delta^{\prime}=\frac{\delta_{0}^{\prime}}{|t|^{n}}$. C $C(t)$ can be chosen similarly as $C(t)=\frac{C_{0}}{|t|^{q}}$. 
The conditions that $\hat{p}_{\Omega}(\phi)<+\infty$, for $\Omega=\Omega(-1, a)$ will give some lower bounds for $m, m^{\prime}, q$; and $m, m^{\prime}, q$, being chosen, the conditions $\hat{p}_{\Omega}(\phi)<\hat{r}$ will give some upper bounds for $\delta_{0}, \delta_{0}^{\prime}, C_{0}$.

Positivity condition in the domain $r<A(t)$. We suppose that the domain $r<A(t)$ is a union of domains $\Omega$. For instance

or

$$
r \leqq \gamma|t|, \text { with } \gamma>1
$$

$$
r \leqq \gamma|t|^{p} \text {, with } \gamma>1, p>1 \text {. }
$$

In such a case the expression of the $T^{A B}$ for $r \leqq A(t)$ does not depend of the exact choice of $A(t)$ and of $\delta^{\prime}(t)$, since for $r \leqq A(t)$, the whole of $D(t, r)$ in (III.8) is also contained in the domain $r \leqq A(t)$.

Choosing $\delta(t)=\frac{\delta_{0}}{|t|^{m}}$, we find:

$$
\begin{aligned}
& T^{00}=\frac{\delta_{0}}{(m-1)(m-2)} \frac{1}{|t|^{m-2}}, \\
& T^{0 i}(t, x)=-\frac{\delta_{0}}{3(m-1)} \frac{x^{i}}{|t|^{m-1}}, \\
& T^{i i}=\frac{\delta_{0}}{(m-1)(m-2)} \frac{1}{|t|^{m-2}}+\frac{\delta_{0}}{6} \frac{r^{2}}{|t|^{m}}-2 C(t), \text { for } i=1, \ldots, 3, \\
& T^{i j}=0 \quad \text { if } \quad i \neq j .
\end{aligned}
$$

Since $T^{i j}=0$, for $i \neq j$, we have

$$
T^{A B} X_{A} X_{B}=T^{00} X_{0}^{2}+2 \sum_{i} T^{0 i} X_{0} X_{i}+\sum_{i} T^{i i} X_{i}^{2}
$$

Putting $\left\|T^{0} \cdot\right\|=\left(\sum_{i}\left(T^{0 i}\right)^{2}\right)^{1 / 2}$, we have, for all $\lambda>0$ :

$$
\begin{gathered}
\left|2 \sum_{i} T^{0 i} X_{0} X_{i}\right| \leqq 2\left|X_{0}\right|\left\|T^{0} \cdot\right\|\left(\sum X_{i}^{2}\right)^{1 / 2} \leqq\left\|T^{0} \cdot\right\|\left(\frac{1}{\lambda} X_{0}^{2}+\lambda \sum X_{i}^{2}\right), \\
T^{A B} X_{A} X_{B} \geqq X_{0}^{2}\left(T^{00}-\frac{1}{\lambda}\left\|T^{0 \cdot}\right\|\right)+\sum_{i} X_{i}^{2}\left(T^{i i}-\lambda\left\|T^{0 \cdot}\right\|\right) .
\end{gathered}
$$

Hence, the positivity condition is verified, if in each point we can choose $\lambda$ such that

$$
\left.\begin{array}{l}
T^{00}-\frac{1}{\lambda}\left\|T^{0} \cdot\right\| \geqq 0 \\
\text { and } T^{i i}-\lambda\left\|T^{0 \cdot}\right\| \geqq 0 \text { for } i=1, \ldots, 3 .
\end{array}\right\}
$$

That is, if

$$
\left\|T^{0 \cdot}\right\|^{2} \leqq T^{00} \cdot T^{i i}
$$

Considering (III.12), we have:

$$
\left\|T^{0} \cdot\right\|=\frac{\delta_{0}}{3(m-1)} \frac{r}{|t|^{m-1}} \leqq \frac{\delta_{0}}{3(m-1)} \frac{A(t)}{|t|^{m-1}} .
$$


Hence

$$
T^{00}-\frac{1}{\lambda}\left\|T^{00}\right\|>\frac{\delta_{0}}{|t|^{m-2}}\left(\frac{1}{(m-1)(m-2)}-\frac{1}{\lambda} \frac{1}{3(m-1)} \cdot \frac{A(t)}{|t|}\right)
$$

That will be positive if $\lambda>\frac{m-2}{3} \frac{A(t)}{|t|}$,

$$
\begin{aligned}
& T^{i i}-\lambda\left\|T^{0}\right\|>-2 C(t)+\frac{\delta_{0}}{|t|^{m-2}}\left(\frac{1}{(m-1)(m-2)}+\frac{1}{6} \frac{r^{2}}{|t|^{2}}-\frac{\lambda r}{3(m-1)|t|}\right), \\
& T^{i i}-\lambda\left\|T^{0}\right\|>-2 C(t)+\frac{\delta_{0}}{|t|^{m-2}}\left(\frac{1}{(m-1)(m-2)}\right. \\
&\left.+\frac{1}{6}\left(\frac{r}{|t|}-\frac{\lambda}{m-1}\right)^{2}-\frac{1}{6} \frac{\lambda^{2}}{(m-1)^{2}}\right)
\end{aligned}
$$

That can be made positive by the choice of $C(t)$, with

$$
C(t)<-\frac{1}{2} \delta_{0} \frac{1}{6(m-1)^{2}}\left(\frac{m-2}{3}\right)^{2}\left(\frac{A(t)}{t}\right)^{2} \frac{1}{|t|^{m-2}} .
$$

For instance, if $A(t)=\gamma|t|$, we can take:

$$
C(t)=-\frac{\delta_{0}}{|t|^{m-2}} \mu, \quad \text { with } \quad \mu>\frac{1}{108}\left(\frac{m-2}{m-1}\right)^{2} .
$$

With a choice of $C(t)$ verifying (III.15), we have an estimate

$$
T^{A B} X_{A} X_{B}>k(t) \sum X_{A}^{2} \text {. }
$$

In the case $A(t)=\gamma|t|$, we have $k(t)=\delta_{0} \frac{k_{0}}{|t|^{m-2}}$, for some $k_{0}>0$.

Positivity Condition in the Domain $r>A(t)$. It may be proved, if we choose $f$ as (III.9) as $r \rightarrow+\infty$,

$$
\begin{gathered}
T^{00}(t, r) \sim \frac{K(t)}{r^{\alpha}}, \\
\left\|T^{0}\right\| \sim\left\{\begin{array}{lll}
\frac{K^{\prime}(t)}{r^{\alpha-1}} & \text { if } & 2<\alpha<3 \\
\frac{K^{\prime}(t)}{r^{2}} & \text { if } & 3<\alpha
\end{array}\right.
\end{gathered}
$$

[That comes from the term $\partial_{j} \phi$ in $g_{j}$, cf. (III.6) and (III.10)]

$$
T^{i i} \sim K^{\prime \prime}(t)
$$

(if we choose $C(t) \neq \frac{\delta}{4} A^{2}+\frac{\delta^{\prime} A^{2-\alpha}}{2(\alpha-2)}$ in (III.10).) In order that the condition (III.13) be verified, we must have:

$$
\begin{aligned}
& \frac{K^{\prime 2}}{r^{2 \alpha-2}}<\frac{K K^{\prime \prime}}{r^{\alpha}} \quad \text { if } \quad 2<\alpha<3 \\
& \frac{K^{12}}{r^{4}}<\frac{K K^{\prime \prime}}{r^{\alpha}} \quad \text { if } \quad 3<\alpha
\end{aligned}
$$


That will be possible only if we choose $2<\alpha<4$. In such a case, with a convenient choice of $C(t)$ it can be proved that the positivity condition may be verified.

However, in such a case, we shall have an estimate

$$
T^{A B} X_{A} X_{B}>k(t, r) \sum X_{A}^{2} .
$$

With $k(t, r)<T^{00}(t, r)$, that means that $k(t, r) \rightarrow 0$ as $r \rightarrow+\infty$. We shall see that such an estimate does not allow us to prove that the solution of exact equations verify also the positivity condition. Therefore we limit ourselves in the search of a solution in a domain $D: r \leqq A(t)$, which is also a union of domains $\Omega$, in such a way that we can apply to $D$ the results we obtained in part II.

Let us give the possible values of $m$ in the simplest case:

$$
D=\{r \leqq \gamma|t|\}=\bigcup_{|a| \leqq \gamma-1} \Omega(-1, a) .
$$

We have found: [cf. (III.15) and (III.10)]

$$
\phi(t, r)=\frac{\delta_{0}}{|t|^{m-2}}\left(-\mu-\frac{1}{12} \frac{r^{2}}{|t|^{2}}\right) .
$$

With such a $\phi$, we have:

$$
\|\phi\|_{\Omega_{t}} \leqq \frac{C \delta_{0}}{|t|^{m-\frac{7}{2}}}, \quad\left\|\nabla^{k} \phi\right\|_{\Omega_{t}} \leqq \frac{C \delta_{0}}{|t|^{m+k-\frac{7}{2}}} .
$$

In order that $\hat{p}_{\Omega}(\phi)<+\infty$, we must choose

$$
m>\frac{19}{2} \text {. }
$$

Remark on the Dominant Energy Condition. Does this solution of approximated equations verify the dominant energy condition? A necessary condition for that is:

$$
T^{00} \geqq\left|T^{0 i}\right| \text {. }
$$

Considering (III.12), (III.19) can be verified only if:

$$
\frac{\delta_{0}}{(m-1)(m-2)}>\frac{\delta_{0}}{3(m-1)} \frac{\left|x^{i}\right|}{|t|},
$$

that means, in the domain $r<\gamma|t|$, if:

$$
m<\frac{3}{\gamma}+2<5, \quad(\text { since } \gamma>1)
$$

Comparing with (III.18), we see that the condition $\hat{p}_{\Omega}(\phi)<+\infty$, implies too great a decay of $\phi$ as $t \rightarrow-\infty$, in order that the dominant energy condition can be verified.

Remark. Another Kind of Solution. We may also have solutions null for $t \leqq t_{0}$ and verifying the positivity condition; taking:

$$
\phi=\left\{\begin{array}{ll}
\left(-r^{2}-b\right) \delta_{0}\left(t-t_{0}\right)^{m} & \text { if } t \geqq t_{0} \\
0 & \text { if } t \leqq t_{0}
\end{array},\right.
$$


it can be proved that if we choose the constant $b$ large enough, the positivity condition is verified and

$$
T^{A B} X_{A} X_{B}>k \delta_{0}\left(t-t_{0}\right)^{m+2} \sum_{A} X_{A}^{2}, \quad \text { with some } k>0 .
$$

B. Positivity Condition for Exact Solutions. Let $\phi^{I J}$ be functions leading to solutions of approximated equations verifying the positivity condition on a domain $D$ which is the union of domains $\Omega$ 's.

The stress-energy tensor of these solutions of approximated equations will be called now $T_{0}=\left(T_{0}^{A B}\right)$, in order to distinguish it from the stress-energy tensor $T=\left(T^{A B}\right)$ of the exact solution with the same data $\phi^{I J}$ on $D$.

We have found solutions such that:

$$
T_{0}^{A B} X_{A} X_{B}>k(t) \sum_{A} X_{A}^{2}, \text { for all vector fields } X_{A} .
$$

We want to show that: $T^{A B} X_{A} X_{B} \geqq 0$ for all timelike vector fields $X_{A}$ - (in fact we shall prove it for all vector fields).

It will be proved, if we show that:

Putting

$$
\left|\left(T^{A B}-T_{0}^{A B}\right) X_{A} X_{B}\right|<T_{0}^{A B} X_{A} X_{B} .
$$

(III.21) will be proved if

$$
\left|T-T_{0}\right|=\left(\sum_{A B}\left|T^{A B}-T_{0}^{A B}\right|^{2}\right)^{1 / 2},
$$

$$
\operatorname{Sup}_{\Omega_{t}}\left|T-T_{0}\right|<k(t)
$$

for all $\Omega$ contained in $D$.

Estimates for $\left|T-T_{0}\right|$. Let $\left(V^{A}, u_{\alpha}^{A}\right)$ be the solution of (I.10) with the data $\phi^{I J}$. Let $\left(V_{0}^{A}\right)$ be the solution of approximated equations (III.3) with the same data $\phi^{I J}$.

From the expression of $T_{0}^{A B}$ in terms of $V_{0}^{A}, \phi^{I J}$ and that of $T^{A B}$ in terms of $\left(V^{A}, \phi^{I J}\right)$ [cf. (III.5)], we deduce an estimate

$$
\left|T-T_{0}\right| \leqq C\left|V-V_{0}\right| \text {. }
$$

Estimates for $\left|V-V_{0}\right|$. If we express the derivatives in the natural frame, the system verified by the $V^{A}$ can be written:

$$
\left\{\begin{array}{l}
\frac{1}{2} \partial_{0} V^{0}+\partial_{i} V^{i}+k^{0}(u) \nabla V=f^{0}(u, V, \phi), \quad \text { where } \quad\left|k^{A}(u)\right|<C|u|, \\
\frac{1}{2} \partial_{j} V^{0}+\partial_{0} V^{j}+k^{j}(u) \nabla V=f^{j}(u, V, \phi) .
\end{array}\right.
$$

The approximated equations verified by the $V_{0}^{A}$, can be written:

$$
\left\{\begin{array}{ll}
\frac{1}{2} \partial_{0} V_{0}^{0}+\partial_{i} V_{0}^{i}=f^{0}(0, V, \phi) & \text { (since for } u=0, \text { terms in } V \\
\frac{1}{2} \partial_{j} V_{0}^{0}+\partial_{0} V_{0}^{j}=f^{j}(0, V, \phi) & \text { disappear in right-hand sides) }
\end{array} .\right.
$$

We deduce, that $V^{A}-V_{0}^{A}$ verify a system:

$$
\left\{\begin{array}{l}
\frac{1}{2} \partial_{0}\left(V^{0}-V_{0}^{0}\right)+\partial_{i}\left(V^{i}-V_{0}^{i}\right)=g^{0}(u, V, \phi) \\
\frac{1}{2} \partial_{j}\left(V^{0}-V_{0}^{0}\right)+\partial_{0}\left(V^{j}-V_{0}^{j}\right)=g^{j}(u, V, \phi)
\end{array},\right.
$$


where $g^{A}(u, V, \phi)=f^{A}(u, V, \phi)-f^{A}(0, V, \phi)-k^{A}(u) \nabla V$, and considering the form of $f^{A}$ [cf. (I.12)], $g$ is of the form:

$$
\begin{aligned}
g(u, V, \phi)= & k(u) \nabla u \otimes \nabla V+k_{1}(u) \nabla V+k(u) \nabla u \otimes \nabla \phi+l_{1}(u) \nabla \phi \\
& \text { with }\left|k_{1}(u)\right|,\left|l_{1}(u)\right|<C|u| .
\end{aligned}
$$

As we have done before, we deduce from (III.24), some estimates:

$$
\left\|V-V_{0}\right\|_{\Omega_{t}} \leqq \int_{-\infty}^{t}\|g\|_{\Omega_{\tau}} d \tau
$$

and considering (III.25), we obtain

$$
\begin{aligned}
\left\|V-V_{0}\right\|_{\Omega_{t}} \leqq & C \int_{-\infty}^{t}\left\{\sum_{k=1}^{3}\left\|\nabla^{k} u\right\|_{\Omega_{\tau}}\left(\|V\|_{\Omega_{\tau}}+\|\phi\|_{\Omega_{\tau}}\right)\right. \\
& \left.+\sum_{k=0}^{2}\left\|\nabla^{k} u\right\|_{\Omega_{\tau}}\left(\|\nabla V\|_{\Omega_{\tau}}+\|\nabla \phi\|_{\Omega_{\tau}}\right)\right\} d \tau .
\end{aligned}
$$

Similarly with the equations derived from (III.24), we obtain estimates for $\left\|\nabla\left(V-V_{0}\right)\right\|_{\Omega_{t}}$ and $\left\|\nabla^{2}\left(V-V_{0}\right)\right\|_{\Omega_{t}}$, and from

we obtain

$$
\operatorname{Sup}_{\Omega_{t}}\left|V-V_{0}\right| \leqq C \sum_{i=0}^{2}\left\|\nabla^{i}\left(V-V_{0}\right)\right\|_{\Omega_{t}},
$$

$$
\begin{aligned}
\operatorname{Sup}\left|V-V_{0}\right| \leqq & \int_{-\infty}^{t}\left\{\sum_{k=0}^{2}\left\|\nabla^{k} u\right\|_{\Omega_{\tau}}\left(\left\|\nabla^{3} V\right\|_{\Omega_{\tau}}+\left\|\nabla^{3} \phi\right\|_{\Omega_{\tau}}\right)\right. \\
& \left.+\sum_{k=0}^{3}\left\|\nabla^{k} u\right\|_{\Omega_{\tau}} \sum_{k=0}^{2}\left(\left\|\nabla^{k} V\right\|+\left\|\nabla^{k} \phi\right\|_{\Omega_{\tau}}\right)\right\} d \tau .
\end{aligned}
$$

Estimates for $\left\|\nabla^{k} u\right\|_{\Omega_{\mathrm{t}}}$ and $\left\|\nabla^{k} V\right\|_{\Omega_{\mathrm{t}}},(k=0, \ldots, 3)$. We have already obtained estimates of $V$ in terms of $\phi$ (cf. II.B.7). We can also obtain estimates of $u$ in terms of $\phi$ :

$u$ verifies the system:

$$
g^{\alpha \beta}(u) \partial_{\alpha \beta} u=\varrho, \quad \text { with } \varrho=-h(u) \nabla u \otimes \nabla u+k(u) V+k(u) \phi .
$$

Then, we have

$$
\|\varrho\|_{\Omega_{t}} \leqq C\left(\|V\|_{\Omega_{t}}+\|\phi\|_{\Omega_{t}}+\operatorname{Sup}_{\Omega_{t}}|\nabla u|\|\nabla u\|_{\Omega_{t}}\right) \text {. }
$$

Then, from (II.A.8) and Gronwall's lemma we deduce an estimate:

$$
\|\nabla u\|_{\Omega_{t}} \leqq C \int_{-\infty}^{t}\left(\|V\|_{\Omega_{\tau}}+\|\phi\|_{\Omega_{\tau}}\right) d \tau
$$

And considering the estimate of $V$, we have

$$
\|\nabla u\|_{\Omega_{t}} \leqq C \int_{-\infty}^{t}(t-\tau+1) \sum_{k=0}^{1}\left\|\nabla^{k} \phi\right\|_{\Omega_{\tau}} d \tau .
$$

From the inequality $\|u\|_{\Omega_{\mathrm{t}}} \leqq C \int_{-\infty}^{t}\|\nabla u\|_{\Omega_{\tau}} d \tau$ and (III.27) we deduce

$$
\|u\|_{\Omega_{t}} \leqq C \int_{-\infty}^{t}(t-\tau+1)^{2} \sum_{k=0}^{1}\left\|\nabla^{k} \phi\right\|_{\Omega_{\tau}} d \tau .
$$


With similar proofs, starting from (II.A.7.8) we obtain estimates

$$
\begin{gathered}
\left\|\nabla^{2} u\right\|_{\Omega_{t}} \leqq C \int_{-\infty}^{t}\left\{(t-\tau+1)^{2}\|\phi\|_{\Omega_{\tau}}+\sum_{k=1}^{2}(t-\tau+1)^{3-k}\left\|\nabla^{k} \phi\right\|_{\Omega_{\tau}}\right\} d \tau, \text { (III.29) } \\
\left\|\nabla^{3} u\right\|_{\Omega_{t}} \leqq C \int_{-\infty}^{t}\left\{(t-\tau+1)^{3}\|\phi\|_{\Omega_{\tau}}+\sum_{k=1}^{3}(t-\tau+1)^{4-k}\left\|\nabla^{k} \phi\right\|_{\Omega_{\tau}}\right\} d \tau \text {. (III.30) }
\end{gathered}
$$

Estimates for $\left|T-T_{0}\right|$ in Terms of $\phi$. From the estimates (III.23, III.26, II.B.7, III.27, 28, 29, 30; we can estimate $\left|T-T_{0}\right|$ in terms of norms of $\phi$ :

$$
\begin{aligned}
\operatorname{Sup}_{\Omega_{\tau}}\left|T-T_{0}\right| \leqq & C \int_{-\infty}^{t} d \tau\left\{\int_{-\infty}^{\tau}\left((\tau-\sigma+1)^{2}\|\phi\|_{\Omega_{\sigma}}+\sum_{k=1}^{3}(\tau-\sigma+1)^{3-k}\|\nabla \phi\|_{\Omega_{\sigma}}\right) d \sigma\right. \\
& \left.\times \int_{-\infty}^{\tau}\left((\tau-\sigma+1)^{3}\|\phi\|_{\Omega_{\sigma}}+\sum_{k=1}^{4}(\tau-\sigma+1)^{4-k}\left\|\nabla^{k} \phi\right\|_{\Omega_{\sigma}}\right) d \sigma\right\} . \text { (III. }
\end{aligned}
$$

Impossibility to conclude on the whole of $V^{T}$. If as $r \rightarrow+\infty, \phi$ does not tend to 0 , the right-hand side in (III.31) with $\Omega=\Omega(T, a)$ does not tend to 0 as $a$ tends to infinity in $\mathbb{R}^{3}$. This estimate does not allow us to conclude that the solution of exact equations verify the positivity condition, since the stress-energy tensor $T_{0}$ of the approximated equations verify an inequality such that (III.16), with $k(t, r) \rightarrow 0$ as $r \rightarrow+\infty$.

Even, if it was possible to choose $C(t)$ in such a way that $\phi \rightarrow 0$ as $r \rightarrow+\infty$ (cf. (II.10) with $\left.C(t)=\frac{\delta}{4} A^{2}+\frac{\delta^{\prime} A^{2-\alpha}}{2(\alpha-2)}\right), \phi$ would tend to 0 at least as $\frac{1}{r}$, therefore the right-hand side of (III.31) would be at least as $\frac{1}{|a|^{2}}$ as $|a| \rightarrow+\infty$, that is the estimate of $\left|T-T_{0}\right|$ would be as $\frac{1}{|a|^{2}}$ as $|a| \rightarrow+\infty$. That would not suffice to conclude since

$$
k(t, r)<T^{00}(t, r) \text { is at most in } \frac{1}{r^{\alpha}}, \text { with } \alpha>2 \text { [cf. (III.11)]. }
$$

Positivity Condition on a Domain D. $D=\{r \leqq A(t)\}$ and $D$ is a union of domains $\Omega$. On such a domain the estimate (III.31) allows to conclude if we make a convenient choice of $\delta(t), A(t)$, and $C(t)$.

Let us give only the result in the case of the instance (III.17). We find

$$
\operatorname{Sup}_{\Omega_{t}}\left|T-T_{0}\right| \leqq \frac{C \cdot \delta_{0}^{2}}{|t|^{2 m-15}} .
$$

We must have

$$
\frac{C \delta_{0}^{2}}{|t|^{2 m-15}}<\frac{k_{0} \delta_{0}}{|t|^{m-2}}
$$

That will be verified if

$$
\left\{\begin{array}{l}
2 m-15>m-2 \Leftrightarrow m>13 \quad \text { and } \\
C \delta_{0}^{2}<k_{0} \delta_{0} \Leftrightarrow \delta_{0}<\frac{k_{0}}{C}
\end{array} .\right.
$$


Conclusion. On a domain $D, r \leqq A(t)$, which is a union of domains $\Omega$, it is possible to find data $\phi^{I J}$ such that the solution of Einstein equations given by the main theorem of part II, and his corollary verify the positivity condition.

An instance is $D=\{r \leqq \gamma|t|\}$

$$
\left\{\begin{array}{l}
\phi^{11}=\phi^{22}=\phi^{33}=\frac{\delta_{0}}{|t|^{m-2}}\left(-\mu-\frac{1}{12} \frac{r^{2}}{|t|^{2}}\right) \\
\phi^{12}=\phi^{23}=\phi^{31}=0
\end{array}\right.
$$

with $\mu>0$ large enough, $m>13$ and $\delta_{0}$ small enough.

Remark. With the other instance given in (III.20), in the same domain $D=\{r \leqq \gamma|t|\}$, using the same method we find:

$$
\operatorname{Sup}_{\Omega_{t}}\left|T-T_{0}\right| \leqq C \delta_{0}^{2}\left(t-t_{0}\right)^{2 m-3}, \quad \text { if } t \geqq t_{0} .
$$

That leads to a solution verifying the positivity condition if $m \geqq 5$ and $\delta_{0}$ small enough.

\section{References}

1. Noutchegueme, N.: Solution des équations d'Einstein avec données minkowskiennes à l'infini passé. Thèse soutenue à l'Université de Yaoundé, 4/3/1986

2. Noutchegueme, N.: Solutions globales des équations d'Einstein pour des sources suffisamment faibles. C.R. Acad. Sci. Paris, t 302, Série I (1986)

3. Noutchegueme, N.: Solutions globales de systèmes quasi linéaires du second ordre. J. Math. Pures Appl. 66, 385-405 (1987)

4. Noutchegueme, N.: Solutions semi-globales asymptotiquement minkowskiennes pour les équations d'Einstein. Annales de l'Inst. Henri Poincaré 46, 77-96 (1987)

5. Cagnac, F., Choquet-Bruhat, Y., Noutchegueme, N.: Solutions of the Einstein equations with data at past-infinity. In: General Relativity and Gravitational Physics. Bruzzo, Cianci, Massa (eds.). Singapore: World Scientific 1987

6. Cagnac, F., Noutchegueme, N.: Solutions globales pour un système quasi-linéaire. C.R. Acad. Sci. Paris, t 306 série I, 365-368 (1988)

7. Cagnac, F., Noutchegueme, N.: Solutions des équations d'Einstein avec sources, minkowskiennes à l'infini passé. C.R. Acad. Sci. Paris 441-444 (1988)

8. Dionne: Sur les problèmes de Cauchy hyperbolique bien posés. J. d'Analyse Math. Jérusalem (1963)

9. Leray: Hyperbolic differential equations. Princeton, NJ: Princeton Univ. Press 1952

10. Choquet-Bruhat, Y.: Systèmes d'équations aux dérivées partielles. Acta Mathematica 88 (1952)

11. Hawking, S., Ellis, G.: The large scale structure of space-time. Cambridge: Camb. Univ. Press (1973)

Communicated by A. Jaffe

Received August 8, 1988; in revised form October 16, 1989 